\title{
1 Linking circadian time to growth rate quantitatively via carbon metabolism
}

3

4

5 Yin Hoon Chew ${ }^{1 \dagger}$, Daniel D. Seaton ${ }^{1}$, Virginie Mengin ${ }^{2}$, Anna Flis $^{2}$, Sam T. Mugford ${ }^{3}$, Alison

6 M. Smith ${ }^{3}$, Mark Stitt ${ }^{2}$, Andrew J. Millar*1

7

8 Affiliations:

$9{ }^{1}$ SynthSys and School of Biological Sciences, C. H. Waddington Building, University of

10 Edinburgh, King's Buildings, Edinburgh EH9 3BF, United Kingdom

$11{ }^{2}$ Max Planck Institute of Molecular Plant Physiology, Am Muehlenberg 1, 14476 Potsdam-

12 Golm, Germany

$13{ }^{3}$ Department of Metabolic Biology, John Innes Centre, Norwich NR4 7UH, United Kingdom

*Corresponding author

Andrew J. Millar

16 Complete address

SynthSys

17 C.H. Waddington Building

18 University of Edinburgh

19 Max Born Crescent

20 Edinburgh EH9 3BF

21 Scotland, UK 
22 Telephone number

: $\quad+44(0) 1316513325$

23 Email

Andrew.Millar@ed.ac.uk

24

25 Current address (YHC) : Department of Genetics \& Genomic Sciences,

26 Icahn Institute for Genomics \& Multiscale Biology, Icahn School of Medicine at Mount Sinai,

271425 Madison Avenue, New York, NY 10029-6574, USA

28

29 Keywords: gene regulatory dynamics; metabolism; genotype to phenotype; mathematical 30 model 
32 Summary paragraph (200 words)

33 Predicting a multicellular organism's phenotype quantitatively from its genotype is 34 challenging, as genetic effects must propagate up time and length scales. Circadian clocks are intracellular regulators that control temporal gene expression patterns and hence metabolism, physiology and behaviour, from sleep/wake cycles in mammals to flowering in plants ${ }^{1-3}$. Clock genes are rarely essential but appropriate alleles can confer a competitive advantage $\mathrm{e}^{4,5}$ and have 38 been repeatedly selected during crop domestication ${ }^{3,6}$. Here we quantitatively explain and predict canonical phenotypes of circadian timing in a multicellular, model organism. We used metabolic and physiological data to combine and extend mathematical models of rhythmic gene expression, photoperiod-dependent flowering, elongation growth and starch metabolism within a Framework Model for growth of Arabidopsis thaliana ${ }^{7-9}$. The model predicted the 43 effect of altered circadian timing upon each particular phenotype in clock-mutant plants. 44 Altered night-time metabolism of stored starch accounted for most but not all of the decrease in whole-plant growth rate. Altered mobilisation of a secondary store of organic acids explained the remaining defect. Our results link genotype through specific processes to higherlevel phenotypes, formalising our understanding of a subtle, pleiotropic syndrome at the wholeorganism level, and validating the systems approach to understand complex traits starting from intracellular circuits. 
51

52

(Text 1507 words)

Small networks of "clock genes" drive 24-hour, biological rhythms in eukaryotic model species $^{1}$. A few among thousands of downstream, clock-regulated genes are known to mediate physiological phenotypes, such as the metabolic syndrome of clock mutant animals ${ }^{10}$. Identifying such causal links cannot predict whole-organism phenotypes quantitatively: formal, mathematical models are required. Predictive modelling in multicellular organisms has best succeeded for phenotypes that closely map the intracellular behaviour of gene circuits ${ }^{11}$, metabolic $^{12}$ or signalling pathways ${ }^{13}$. Circadian clocks in contrast integrate multiple environmental inputs and affect disparate, potentially interacting biological processes, up to organismal growth and lifecycle traits ${ }^{4,14}$. Mis-timed mutant organisms suffer a syndrome of mild, environment-dependent effects akin to a chronic disease ${ }^{1,4,10}$.

The Arabidopsis clock mechanism ${ }^{1}$ comprises dawn-expressed transcription factors LATE ELONGATED HYPOCOTYL (LHY) and CIRCADIAN CLOCK-ASSOCIATED 1 (CCA1), which inhibit the expression of evening genes such as GIGANTEA (GI) (Fig.1a). LHY and CCA1 expression is inhibited by PSEUDO-RESPONSE REGULATOR (PRR) proteins. Removing the earliest-expressed $P R R$ genes in $\operatorname{prr} 7$ prr 9 mutants slows the clock $^{15}$ by delaying the decline of $L H Y$ and CCAl expression and the subsequent rise of their targets (Fig.1b). Mathematical models of this circuit ${ }^{16}$ have been extended to intermediate transcription factors, including factors that regulate flowering time and organ elongation ${ }^{7}$. We therefore tested whether these causal links were sufficient to understand (explain and predict) the multiple phenotypes of a clock mutant genotype.

The Arabidopsis Framework Model $(\mathrm{FMv} 1)^{9}$ represents the interacting physiological components of whole-organism phenotypes, in a simple, modular fashion. Flowering time in Arabidopsis is commonly scored by the number of rosette leaves, for example. Predicting leaf number involves the FM's clock and photoperiod ${ }^{7}$, phenology ${ }^{17}$ and functional-structural sub- 
models ${ }^{18}$. Adding a clock sub-model that explicitly represents $P R R 7, P R R 9$ and output pathways (see Supplementary Methods; Fig.2) was sufficient to match the published, lateflowering phenotype ${ }^{19}$ of $\operatorname{prr}$ prr9 compared to wild-type Columbia (Col) plants under long photoperiods (Fig.1c). Under short photoperiods, the mutant phenotype is weaker (Extended Data Fig.1a). The model also matched the observed ${ }^{20}$, photoperiodic regulation of hypocotyl elongation in wild-type plants and qualitatively matched the longer hypocotyls of prr7prr9 (Extended Data Fig.1b).

Biomass growth is mediated by the metabolic network, the development of sink and source organs and resource partitioning amongst them. Here, we test the importance of one of many potential circadian effects on biomass, via the nightly, clock-limited rate of sugar mobilisation from storage in transient $\operatorname{starch}^{21}$. To understand these carbon dynamics in prr7prr9, we first extended the metabolic sub-model. Daytime starch accumulation in wild-type plants under short photoperiods was underestimated in the $\mathrm{FMv}^{9,22}$. Partitioning of photoassimilate towards starch in the model was therefore updated using the measured activity of the key biosynthetic enzyme, AGPase, which partitions more carbon to starch under short photoperiods than is allowed for in the FMv1 (Supplementary Methods; Extended Data Fig.2a). At night, starch is mobilised (degraded) at a constant rate to provide sugar until dawn, as anticipated by the circadian clock $^{21,23}$. We therefore linked the starch degradation rate to the clock sub-model ${ }^{8}$

94 (Supplementary Methods). Simulation of the revised model closely matched end-of-day starch levels under photoperiods of $12 \mathrm{~h}$ or less (Fig.1e). Finally, the organic acids malate and fumarate also accumulate significantly during the day in Arabidopsis, are mobilised at night and have

97 been proposed as secondary carbon stores $^{24}$. At the end of the day, levels of malate and fumarate were two-fold higher in $\operatorname{prr} 7 \operatorname{prr} 9$ than wild-type, with a smaller elevation of citrate, aconitate and iso-citrate (Figs.1d, Extended Data Fig.3). Malate and fumarate were therefore 100 included as an organic acid pool with dynamics similar to starch, in an extended model termed 
the FMv2 (Fig.2). The FMv2 predicts the gain of carbon biomass directly and other major biomass components indirectly. For example, the 3.3-fold increase in protein synthesis rates from night to day predicted by the model was very close to the observed 3.1 -fold increase ${ }^{25}$ (see Supplementary Methods). If altered starch mobilisation in the clock mutant was sufficient to affect its biomass, the FMv2 should also predict that phenotype.

We first tested whether the FMv2 could explain the phenotypes caused by a direct change in starch degradation, in mutants of LIKE SEX FOUR 1 (LSF1). LSF1 encodes a phosphatase homologue necessary for normal starch mobilisation ${ }^{26}$. lsfl mutants grown under 12L:12D have mildly elevated starch levels and reduced biomass ${ }^{26}$, similar to the $\operatorname{prr} 7 \mathrm{prr} 9$ clock mutant

110 (Fig.3b). Reducing the relative starch degradation rate alone in the FMv2 recapitulated the lsf1 111 starch excess observed in published studies ${ }^{26}$ (Extended Data Fig.1c) and new datasets

112 (Figs.3g,3i). The higher baseline starch level arises naturally if the plant is close to a steady 113 state, where the absolute amount of starch degraded nightly in $l s f l$ equals the daily synthesis.

114 Absolute starch synthesis in $l s f 1$ is wild-type (Fig.3g,3i). To degrade the same amount of starch 115 as wild-type at a lower relative rate, the $l s f 1$ mutant must have a higher baseline starch level.

116 The assumption of a lower relative degradation rate in $l s f l$ is therefore functionally equivalent

117 to but conceptually simpler than the previous assumption of an altered 'starch set point' 118 baseline level ${ }^{23,26}$.

119 A minimal model calibration workflow (Extended Data Fig.4) allowed comparison of simulations of the FMv2 with measurements from multiple experiments on $\operatorname{prr} 7 \operatorname{prr} 9$ and $l s f 1$ mutants. Measured photosynthetic and metabolic variables (Extended Data Fig.5) calibrated up to 4 model parameters (Extended Data Table 1), and the genotype-specific water content ${ }^{9}$.

123 Reducing the relative starch degradation rate in the calibrated model accurately predicted the 124 reduced biomass of $l_{s f l}$ mutant plants in each case (Figs.3c,3e), despite the apparent paradox that the mutants mobilised the same absolute amount of starch as the wild type. The explanation 
supported by the model is that $l s f 1$ mutant plants accumulate large, unused starch pools as well as new biomass, whereas wild-type plants produce biomass more efficiently, leaving only a minimum of carbon in starch. The coefficient of variation of the Root-Mean-Square Error (cvRMSE) provides a normalised error metric for all biomass data ${ }^{9}$, showing a good fit to both $130 \quad l s f 1$ and wild-type genotypes $\left(10.1 \%, 15.3 \% \mathrm{Col}\right.$ and $13.7 \%, 15.4 \% l_{s} f 1$ in experiments 1 and 2 respectively). Altering the relative starch degradation rate therefore explained both the $l s f 1$ mutant's modest starch excess and its reduced biomass, validating the model.

prr7prr9 mutants showed slower relative starch degradation (Fig.3a) and higher starch levels at both dawn and dusk (Extended Data Fig.1d) than the wild type. Simulating prr7prr9 mutations in the clock sub-model matched these phenotypes for plants grown in Norwich (Figs.3a, Extended Data 1e) and Edinburgh (Fig.3h), indicating that the mutant clock's later estimate of subjective dawn explained the starch degradation defect. $\operatorname{prr}^{7}$ single mutants ${ }^{27}$ fully mobilised starch and grew normally, as predicted (Extended Data Fig.6). Although model calibration data showed that photosynthesis, starch synthesis and leaf production rates were unaffected by the mutations (Extended Data Fig.5), biomass of prr7prr9 mutant plants was strongly reduced relative to wild-type plants in independent studies (by $40 \%$ and $31 \%$ at 38 days in experiments 1 and 2 respectively). However, the calibrated FMv2 predicted much smaller reductions in biomass in prr7prr9 due to accumulating starch $(26 \%$ and $18 \%$ in

144 experiments 1 and 2 respectively). Neither 1 S.D. variation in the mutant's simulated water content, the most sensitive parameter in our model (Extended Data Fig.7), nor any measured water content value allowed the model with only a starch defect to match the mutant biomass

147 (Extended Data Fig.8). The poor model fits (cvRMSE $=41 \%, 45 \%$ in experiments 1 and 2 148 respectively) indicated that process(es) additional to starch degradation limited the growth of 149 prr7prr9 but not of $l s f 1$ plants. 
150 Considering malate and fumarate as a secondary carbon store $^{24}$, the amount of carbon

151 mobilised from malate and fumarate at night in the wild type was up to $19 \%$ of the carbon

152 mobilised from starch. prr7prr9 but not $l s f 1$ plants accumulated excess malate and fumarate,

153 representing further 'wasted' carbon that did not contribute to subsequent growth (Figs.3k-n).

154 We therefore reduced the relative malate and fumarate mobilisation rate in the FMv2

155 simulation of $\operatorname{prr} 7$ prr9, to reproduce the observed organic acid excess (Figs.31,3n). Together,

156 the simulated defects in starch and organic acid mobilisation quantitatively accounted for the

157 mutant's reduced biomass (Figs.3d,3f; cvRMSE $=14.4 \%, 20.1 \%$ in experiments 1 and 2

158 respectively).

159 The FMv2 built upon delayed gene expression patterns in $\operatorname{prr} 7$ prr 9 mutants to predict canonical

160 clock phenotypes: altered hypocotyl elongation, flowering time, starch metabolism and hence

161 most (58-65\%) of the mutants' reduced biomass. Unused malate and fumarate accounted for

162 their remaining biomass defect, and might similarly affect arrhythmic prr 5 prr 7 prr 9 mutants ${ }^{28}$.

163 Carbon supply limited growth in our well-watered, nutrient-rich growth conditions ${ }^{22}$, though

164 carbon limitation was milder than in conditions that reduced the chlorophyll content of clock mutants $^{4}$ or triggered sugar signals to alter timing ${ }^{27}$. Future extensions of the model could address the nutrient and water limitations that prevail in field conditions, test further aspects of

167 circadian regulation and critical functions of plant biology with daily regulation, such as

168 photosynthesis. Our results suggest a broader proof of principle, that the contributions of

169 dynamic gene regulation and metabolism to whole-organism physiology will also be

170 understood (explained and predicted) quantitatively in other multicellular species ${ }^{29}$, for

171 example using clock and metabolic models in animals and humans to understand body

172 composition $^{10}$. 
Acknowledgements

175 Supported by European Commission FP7 collaborative project TiMet (contract 245143) and

176 by a BBSRC Institute Strategic Programme Grant BB/J004561/1 to the John Innes Centre.

178 Author Contributions

179 YHC, AS, MS and AJM designed the study. YHC, VM, AF, SM, AS and MS performed the experiments and analysed the experimental data. YHC and DDS performed the modelling and analysed the simulation results. YHC, DDS and AJM wrote the paper with input from all authors.

\section{Figure legends}

185 Figure 1: Simulation of clock dynamics and clock outputs.

186 (a,b) Clock gene mRNA abundance ${ }^{30}$ for wild-type (Col) and prr7prr9 plants (dashed lines, 187 symbols), and FMv2 simulations (solid lines), under 12h light:12h dark cycles (12L:12D),

188 double-plotted, normalised to Col level. (c) Rosette leaf number at flowering ${ }^{19}$ under 16L:8D

189 (filled), compared to simulation (open). (d) Malate and fumarate accumulation (mean \pm SEM, $190 \mathrm{n}=4)$ in Col and prr7prr9 at end of day (ED) or night (EN) under 12L:12D, 20 ${ }^{\circ} \mathrm{C}$, light 191 intensity $=160 \mu \mathrm{mol} / \mathrm{m}^{2} / \mathrm{s}$; t-tests compared $\operatorname{prr} 7 p r r 9$ to $\operatorname{Col}(* \mathrm{p}<0.05 ; * * * \mathrm{p}<0.001)$. (e) Starch

192 levels at ED (filled) and EN (open) after 30 days under various photoperiods ${ }^{22}$ (triangles), 193 compared to FMv1 (squares), FMv2 (circles). 
Figure 2: Schema of the Framework Model.

197 The FMv2 includes a clock gene circuit sub-model (upper section). Clock outputs (red arrows)

198 regulate hypocotyl elongation via the PIF components, flowering via FT mRNA production

199 and starch degradation via the timer T. Environmental inputs affect multiple model components

200 (shaded). Vegetative growth is driven by the positive feedback on photosynthesis, mediated by

201 sugar-powered growth of photosynthetically active leaf area within the plant structure. The

202 FMv2 includes nightly carbon storage both in starch and in a secondary, organic acid pool,

203 comprising malate and fumarate (Mal+Fum). Components tested by mutation (PRR9, PRR7,

204 LSF1) are shown in red (see Extended Data).

206 Figure 3: Contributions of starch and organic acids to biomass growth.

207 (a) $\operatorname{prr} 7 \operatorname{prr} 9$ (blue, squares) mobilised starch more slowly than Col (green, circles); normalised 208 to Col peak (mean \pm SEM, n=6). (b) 38-day-old Col, lsf1, prr7prr9. (c-n) Data (symbols) and

209 simulation (lines) of fresh weight (c-f), starch (g-j) and total malate and fumarate (k-n) for Col 210 (circles, green), $l s f 1$ (triangles, orange) and $\operatorname{prr} 7 \operatorname{prr} 9$ (squares; dashed black, simulation of 211 starch defect; blue, starch and organic acid defects). (d,f) Insets enlarge main panel. Data show 212 mean $\pm \mathrm{SD} ; \mathrm{n}=5$ for biomass; $\mathrm{n}=3$ for metabolites, where each sample pooled 3 plants. 213 Temperature $=20^{\circ} \mathrm{C}$ (a), $20.5^{\circ} \mathrm{C}$ (b, Experiment1), $18.5^{\circ} \mathrm{C}$ (Experiment2); 12L:12D light

214 intensity $=190 \mu \mathrm{mol} / \mathrm{m}^{2} / \mathrm{s}(\mathrm{a}), 145 \mu \mathrm{mol} / \mathrm{m}^{2} / \mathrm{s}(\mathrm{b}-\mathrm{n}) ; \mathrm{CO}_{2}=420 \mathrm{ppm}$. 


\section{Methods}

\section{Experimental methods}

\section{Plant materials and growth conditions}

218 Arabidopsis thaliana of the Columbia (Col-0) accession, $p r r 7-3 / p r r 9-1^{19}$ and $l s f 1-1^{26}$ were used

219 in this study. Seeds were first sown on half strength Murashige and Skoog (MS) solution and 220 stratified in darkness at $4^{\circ} \mathrm{C}$ for 5 days before being exposed to white light at the desired

221 photoperiod and temperature. Four-day-old seedlings were then transferred to soil containing

222 Levington seed and modular compost (plus sand). The growth and treatment conditions for each experiment are shown in the figure legends. For the experiment in Fig.1d and Extended

224 Data Fig.3 only, seeds were sown on wet soil in pots and transferred directly to experimental conditions. Plants were thinned after a week and treated with Nematodes after two weeks as a biological pest control.

\section{Leaf number and plant assay}

The total number of leaves (including the cotyledons) was recorded every 3-4 days from seedling emergence. Only leaves exceeding $1 \mathrm{~mm}^{2}$ in size (by eye) were considered in the total leaf count. Plants were harvested for biomass at different time points and for metabolite measurement at 3 weeks (Extended Data Fig.3) and 4 weeks (other data). For metabolite measurement, rosettes were harvested and immediately submerged in liquid nitrogen, half an hour before lights off (end of day, ED) or lights on (end of night, EN) and stored at $-80^{\circ} \mathrm{C}$ until extraction. For dry biomass, dissected plants were oven-dried at $80^{\circ} \mathrm{C}$ for 7 days. Area analysis isolate the green region, which was next converted into binary format. The area was then

237 determined using the Analyze Particles tool. 
239 Gas exchange measurement

240 An EGM-4 Environmental Gas Monitor for $\mathrm{CO}_{2}$ (PP Systems, US) was used for $\mathrm{CO}_{2}$ flux

241 measurement. A Plexiglass cylindrical chamber $(12 \mathrm{~cm}$ in diameter $\mathrm{x} 3 \mathrm{~cm}$ sealed height, with

242 a $6 \mathrm{~cm}$ tall support) was used (Extended Data Fig.5f). Rubber rings around the lid and the hole

243 for the pot ensured an airtight seal. The chamber was connected to the EGM-4 with two butyl

244 tubes for closed-loop measurement.

245 Each individual measurement was taken by placing an individual plant pot in the chamber for

246 approximately 60 seconds, during which the EGM-4 recorded $\mathrm{CO}_{2}$ concentration $\left(\mu \mathrm{mol} \mathrm{mol}{ }^{-1}\right.$

247 or ppm) every 4.6 seconds. We covered the soil surface of the pots with black opaque plastic,

248 leaving only a small hole in the middle for the plants. Plants were measured when they were

24937 days old. Dark respiration was measured one hour before lights-on while daytime

250 assimilation was measured one hour before lights-off.

$251 \mathrm{CO}_{2}$ enrichment of the atmosphere in the growth chambers due to the experimenters' breathing was avoided by using a breath-scrubbing device during measurement. Hourly $\mathrm{CO}_{2}$ concentration at leaf level was also monitored by connecting the EGM-4 to a computer for automated data logging. The average hourly $\mathrm{CO}_{2}$ level was used as input to the model.

\section{Extraction and determination of metabolite content}

256 Rosettes were harvested as described above and ground in liquid nitrogen. Around 20mg of

257 ground material was aliquoted in screw-cap tubes (Micronic). Ethanolic extraction was 258 performed using $80 \%$ ethanol v/v with $10 \mathrm{mM}$ MES (pH 5.9) and 50\% ethanol v/v with $10 \mathrm{mM}$ 259 MES (pH 5.9). During extraction, the successive supernatants obtained were combined into 260 96-deep well plates. The supernatant was used for spectrophotometric determination of 261 chlorophylls, soluble carbohydrates, amino acids and organic acids as described ${ }^{32}$. The pellet 
262 remaining after the ethanolic extraction was used for the determination of starch and total

263 protein content as described ${ }^{33}$.

\section{Modelling methods}

265 Development of the FMv2 in Matlab (Mathworks, Cambridge, UK), model equations, 266 experimental data for model calibration and simulation procedures are described in the

267 Supplementary Methods section.

\section{Data and model availability}

269 A simulator to run the FMv2 in multiple conditions is publicly accessible online at 270 http://turnip.bio.ed.ac.uk/fm/. Numerical data and model files will be available from the

271 University of Edinburgh DataShare www.datashare.ed.ac.uk [insert doi].

\section{References}

2741 Millar, A. J. The intracellular dynamics of circadian clocks reach for the light of 275 ecology and evolution Annu Rev Plant Biol 67, 595-618, doi:10.1146/annurev-arplant$276 \quad$ 043014-115619 (2016).

2772 Bass, J. \& Takahashi, J. S. Circadian integration of metabolism and energetics. Science 278 330, 1349-1354 (2010).

2793 Bendix, C., Marshall, C. M. \& Harmon, F. G. Circadian Clock Genes Universally 280 Control Key Agricultural Traits. Mol Plant 8, 1135-1152, $281 \quad$ doi:10.1016/j.molp.2015.03.003 (2015).

2824 Dodd, A. N. et al. Plant circadian clocks increase photosynthesis, growth, survival, and competitive advantage. Science 309, 630-633, doi:10.1126/science.1115581 (2005). 
2845 Ouyang, Y., Andersson, C. R., Kondo, T., Golden, S. S. \& Johnson, C. H. Resonating circadian clocks enhance fitness in cyanobacteria. Proc Natl Acad Sci U S A 95, 86608664 (1998).

2876 Muller, N. A. et al. Domestication selected for deceleration of the circadian clock in $288 \quad$ cultivated tomato. Nat Genet, doi:10.1038/ng.3447 (2015).

2897 Seaton, D. D. et al. Linked circadian outputs control elongation growth and flowering in response to photoperiod and temperature. Mol Syst Biol 11, 776, doi:10.15252/msb.20145766 (2015).

Seaton, D. D., Ebenhoh, O., Millar, A. J. \& Pokhilko, A. Regulatory principles and experimental approaches to the circadian control of starch turnover. $J R$ Soc Interface 11, 20130979, doi:10.1098/rsif.2013.0979 (2014).

Chew, Y. H. et al. Multiscale digital Arabidopsis predicts individual organ and wholeorganism growth. Proc Natl Acad Sci $U$ S A 111, E4127-4136, doi:10.1073/pnas.1410238111 (2014).

29810 Peek, C. B. et al. Circadian clock NAD+ cycle drives mitochondrial oxidative metabolism in mice. Science 342, 1243417, doi:10.1126/science.1243417 (2013).

30011 von Dassow, G., Meir, E., Munro, E. M. \& Odell, G. M. The segment polarity network is a robust developmental module. Nature 406, 188-192 (2000).

30212 Grafahrend-Belau, E. et al. Multiscale metabolic modeling: dynamic flux balance 303 analysis on a whole-plant scale. Plant Physiol 163, 637-647, 304 doi:10.1104/pp.113.224006 (2013).

30513 Band, L. R. et al. Systems analysis of auxin transport in the Arabidopsis root apex. Plant Cell 26, 862-875, doi:10.1105/tpc.113.119495 (2014).

30714 Green, R. M., Tingay, S., Wang, Z. Y. \& Tobin, E. M. Circadian rhythms confer a higher level of fitness to Arabidopsis plants. Plant Physiol 129, 576-584. (2002). 
30915 Nakamichi, N. et al. PSEUDO-RESPONSE REGULATORS 9, 7, and 5 are

310 transcriptional repressors in the Arabidopsis circadian clock. Plant Cell 22, 594-605

$311 \quad(2010)$.

31216 Pokhilko, A. et al. The clock gene circuit in Arabidopsis includes a repressilator with 313 additional feedback loops. Mol Syst Biol 8, 574, doi:10.1038/msb.2012.6 (2012).

31417 Chew, Y. H. et al. An augmented Arabidopsis phenology model reveals seasonal 315 temperature control of flowering time. New Phytol 194, 654-665, doi:10.1111/j.1469-

$316 \quad$ 8137.2012.04069.x (2012).

31718 Christophe, A. et al. A model-based analysis of the dynamics of carbon balance at the 318 whole-plant level in Arabidopsis thaliana. Functional Plant Biology 35, 1147-1162, 319 doi:Doi 10.1071/Fp08099 (2008).

32019 Nakamichi, N. et al. Arabidopsis clock-associated pseudo-response regulators PRR9, PRR7 and PRR5 coordinately and positively regulate flowering time through the canonical CONSTANS-dependent photoperiodic pathway. Plant Cell Physiol 48, 822832, doi:10.1093/pcp/pcm056 (2007).

32420 Niwa, Y., Yamashino, T. \& Mizuno, T. The circadian clock regulates the photoperiodic response of hypocotyl elongation through a coincidence mechanism in Arabidopsis thaliana. Plant Cell Physiol 50, 838-854 (2009).

32721 Graf, A., Schlereth, A., Stitt, M. \& Smith, A. M. Circadian control of carbohydrate availability for growth in Arabidopsis plants at night. Proc Natl Acad Sci U S A 107,

33022 Sulpice, R. et al. Arabidopsis coordinates the diurnal regulation of carbon allocation and growth across a wide range of photoperiods. Mol Plant 7, 137-155, doi:10.1093/mp/sst127 (2014). 
33323 Scialdone, A. et al. Arabidopsis plants perform arithmetic division to prevent starvation

334 at night. Elife 2, e00669, doi:10.7554/eLife.00669 (2013).

33524 Zell, M. B. et al. Analysis of Arabidopsis with highly reduced levels of malate and 336 fumarate sheds light on the role of these organic acids as storage carbon molecules.

337 Plant Physiol 152, 1251-1262, doi:10.1104/pp.109.151795 (2010).

33825 Ishihara, H., Obata, T., Sulpice, R., Fernie, A. R. \& Stitt, M. Quantifying Protein 339 Synthesis and Degradation in Arabidopsis by Dynamic (CO2)-C-13 Labeling and 340 Analysis of Enrichment in Individual Amino Acids in Their Free Pools and in Protein 341 (vol 168, pg 74, 2015). Plant Physiol 168, 1179-1179, doi:10.1104/pp.15.00771 (2015).

34226 Comparot-Moss, S. et al. A putative phosphatase, LSF1, is required for normal starch turnover in Arabidopsis leaves. Plant Physiol 152, 685-697, doi:10.1104/pp.109.148981 (2010).

34527 Haydon, M. J., Mielczarek, O., Robertson, F. C., Hubbard, K. E. \& Webb, A. A. Photosynthetic entrainment of the Arabidopsis thaliana circadian clock. Nature 502, 689-692, doi:10.1038/nature12603 (2013).

34828 Fukushima, A. et al. Impact of clock-associated Arabidopsis pseudo-response regulators in metabolic coordination. P Natl Acad Sci USA 106, 7251-7256, doi:10.1073/pnas.0900952106 (2009).

29 Le Novere, N. Quantitative and logic modelling of molecular and gene networks. Nat Rev Genet 16, 146-158, doi:10.1038/nrg3885 (2015).

30 Flis, A. et al. Defining the robust behaviour of the plant clock gene circuit with absolute RNA timeseries and open infrastructure. Open Biol 5, doi:10.1098/rsob.150042 (2015). 
35831 Schneider, C. A., Rasband, W. S. \& Eliceiri, K. W. NIH Image to ImageJ: 25 years of

359 image analysis. Nat. Meth. 9, 671-675, doi:Doi 10.1038/Nmeth.2089 (2012).

36032 Arrivault, S. et al. Use of reverse-phase liquid chromatography, linked to tandem mass

361 spectrometry, to profile the Calvin cycle and other metabolic intermediates in

362 Arabidopsis rosettes at different carbon dioxide concentrations. Plant J 59, 826-839,

363 doi:10.1111/j.1365-313X.2009.03902.x (2009).

36433 Pyl, E. T. et al. Metabolism and growth in Arabidopsis depend on the daytime 365 temperature but are temperature-compensated against cool nights. The Plant cell 24, $366 \quad$ 2443-2469, doi:10.1105/tpc.112.097188 (2012). 

available under aCC-BY 4.0 International license.

a

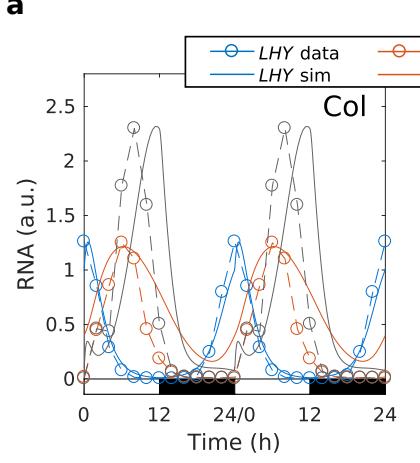

C

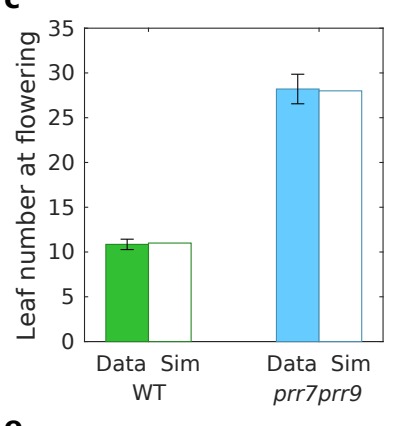

d Malate Fumarate
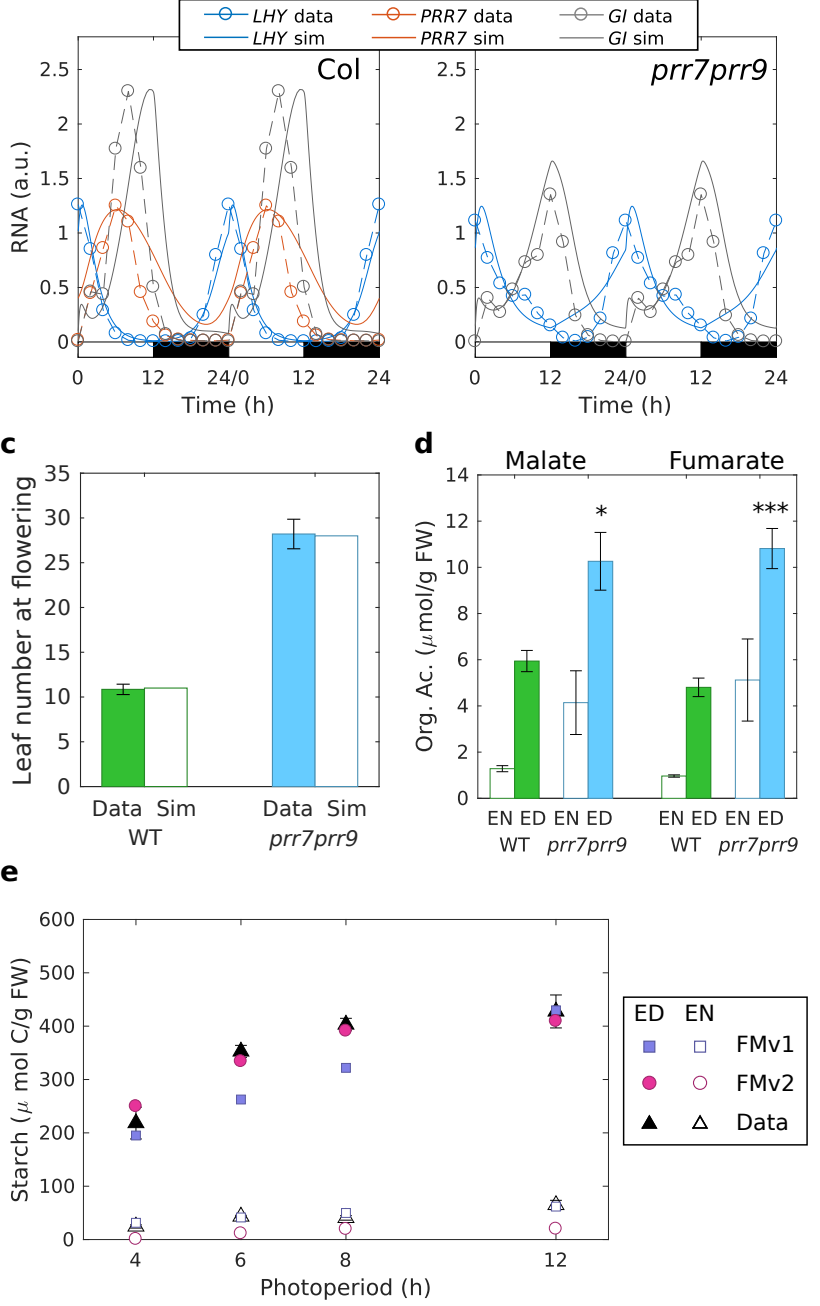

Figure 1: Simulation of clock dynamics and clock outputs.

$(a, b)$ Clock gene mRNA abundance ${ }^{30}$ for wild-type (Col) and prr7prr9 plants (dashed lines, symbols), and FMv2 simulations (solid lines), under $12 \mathrm{~h}$ light:12h dark cycles (12L:12D), double-plotted, normalised to Col level. (c) Rosette leaf number at flowering ${ }^{19}$ under 16L:8D (filled), compared to simulation (open). (d) Malate and fumarate accumulation (mean \pm SEM, $n=4$ ) in Col and prr7prr9 at end of day (ED) and end of night (EN) under $12 \mathrm{~L}: 12 \mathrm{D}, 20^{\circ} \mathrm{C}$, light intensity $=160 \mu \mathrm{mol} / \mathrm{m}^{2} / \mathrm{s}$; t-tests compared prr7prr9 to Col (* $\mathrm{p}<0.05$; *** $\mathrm{p}<0.001$ ). (e) Starch levels at ED (filled) and EN (open) after 30 days under various photoperiods ${ }^{22}$ (triangles), compared to FMv1 (squares), FMv2 (circles). 

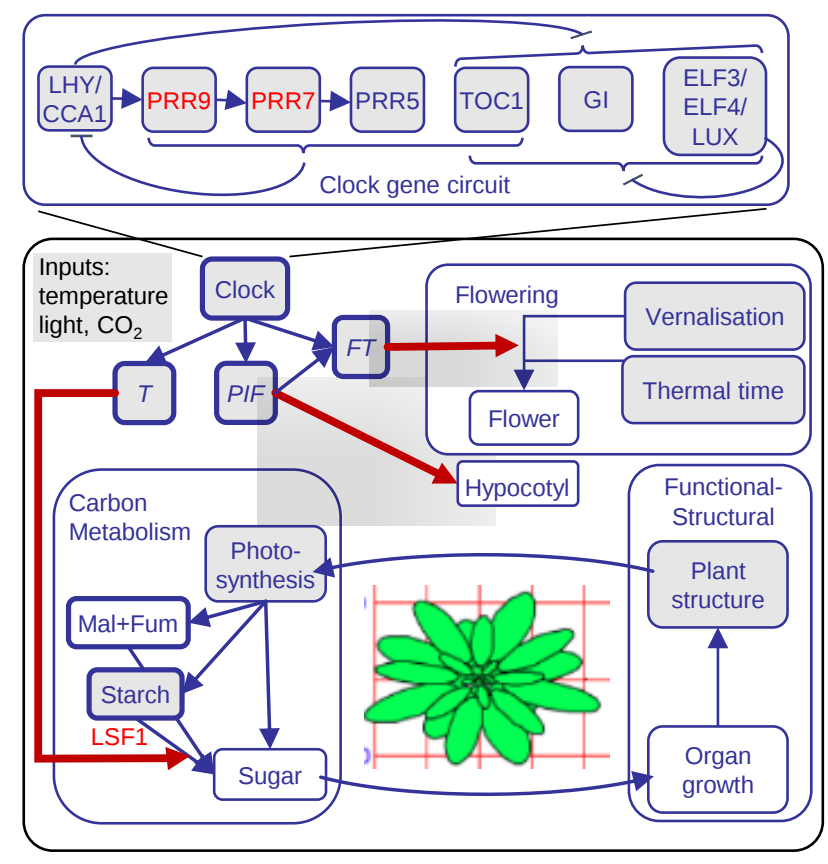

Figure 2: Schematic of the Framework Model.

The FMv2 includes a clock gene circuit sub-model (upper section). Clock outputs (red arrows) regulate hypocotyl elongation via the PIF components, flowering via FT mRNA production and starch degradation via the timer $T$. Environmental inputs affect multiple model components (shaded). Vegetative growth is driven by the positive feedback on photosynthesis, mediated by sugar-powered growth of photosynthetically active leaf area within the plant structure. The FMv2 includes nightly carbon storage both in starch and in a secondary, organic acid pool, comprising malate and fumarate (Mal+Fum). Components tested by mutation (PRR9, PRR7, LSF1) are shown in red (see Extended Data). 


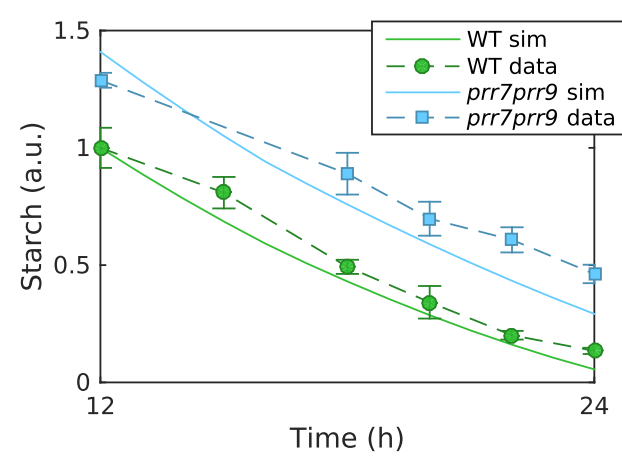

Experiment 1
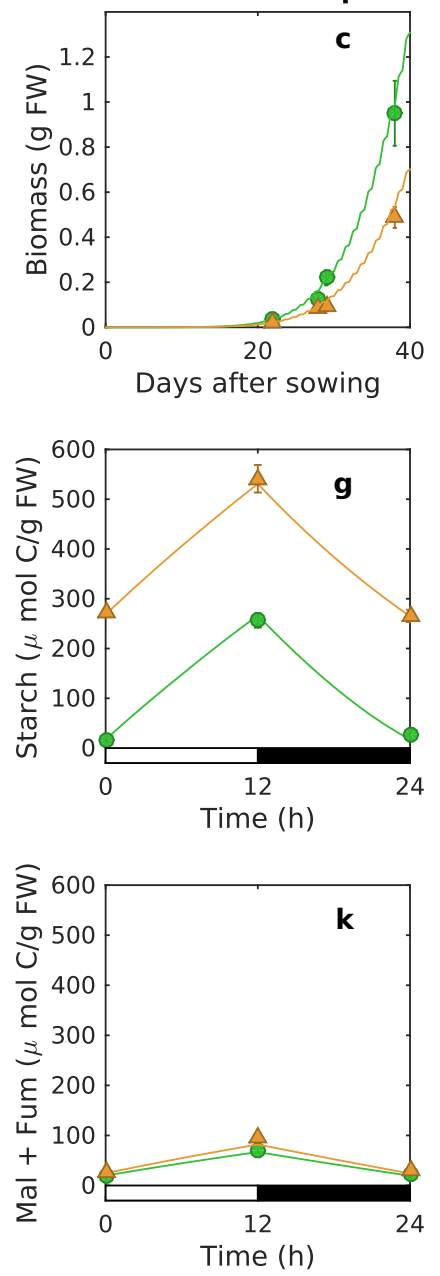
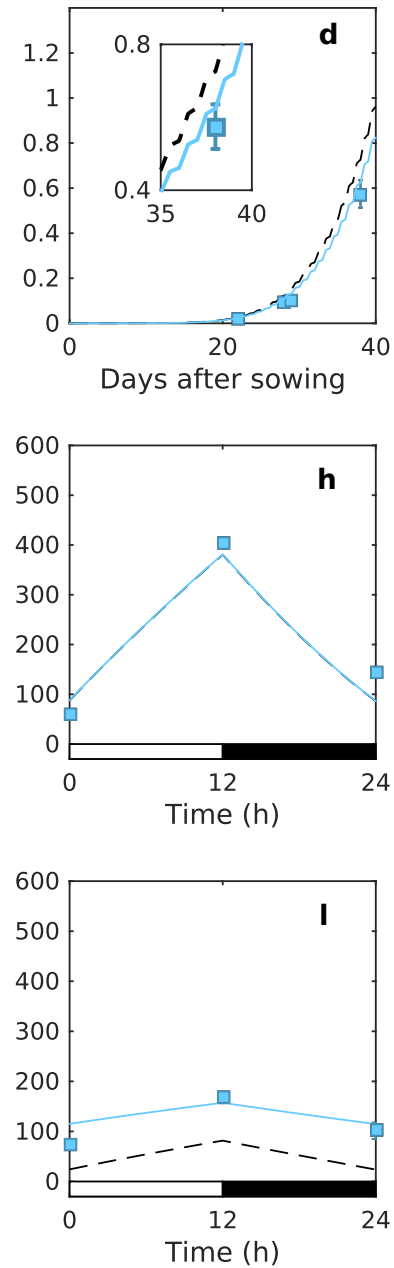

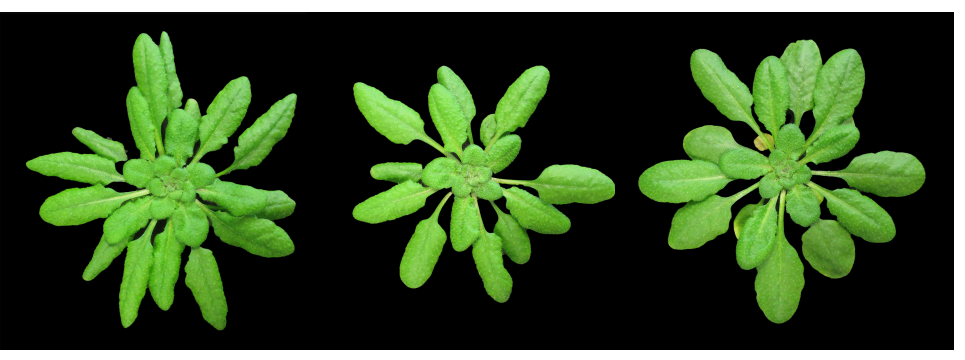

$\mathrm{Col}$

prr7prr9
Experiment 2
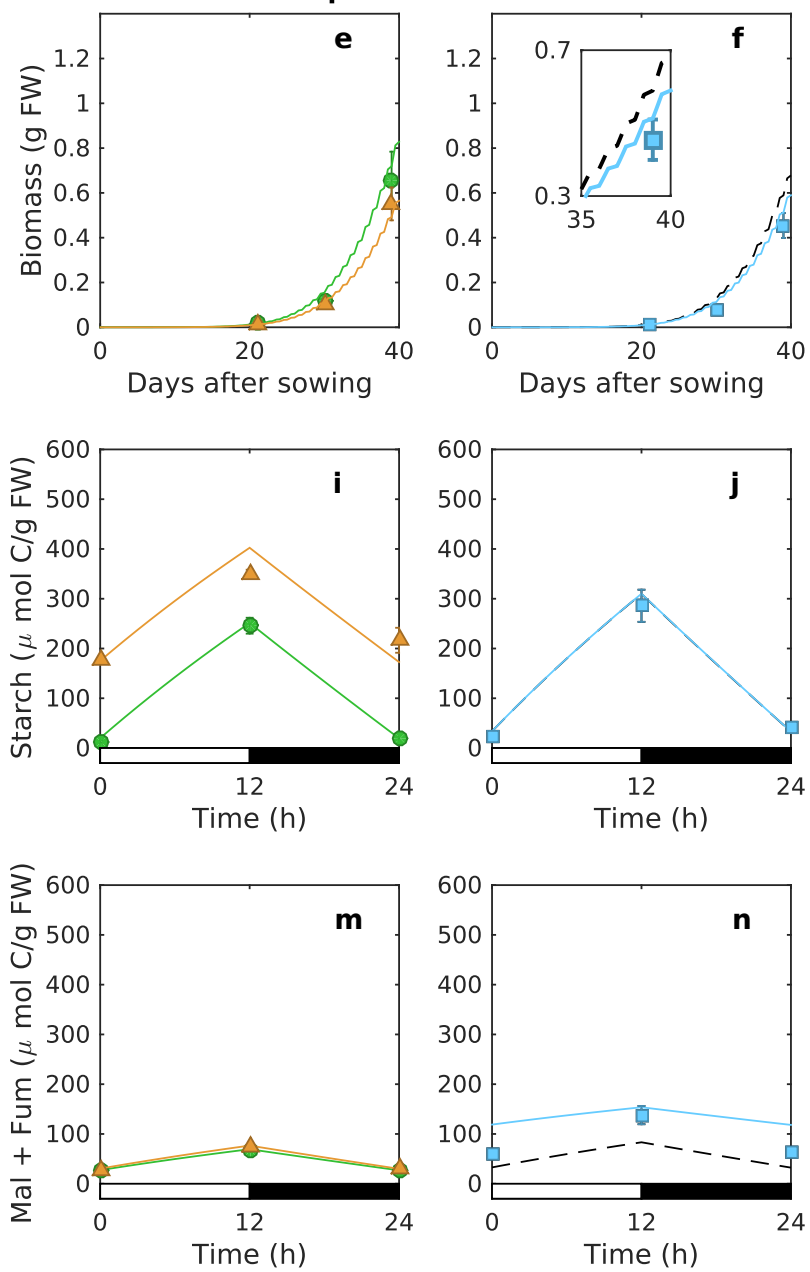

Figure 3: Contributions of starch and organic acids to biomass growth.

(a) prr7prr9 (blue, squares) mobilised starch more slowly than Col (green, circles); normalised to Col peak (mean \pm SEM, $n=6$ ). (b) 38-day-old Col, Isf1, prr7prr9. (c-n) Data (symbols) and simulation (lines) of fresh weight (c-f), starch ( $g$-j) and total malate and fumarate (k-n) for Col (circles, green), Isf1 (triangles, orange) and prr7prr9 (squares; dashed black, simulation of starch defect; blue, starch and organic acid defects). (d,f) Insets enlarge main panel. Data show mean $\pm S D ; n=5$ for biomass; $n=3$ for metabolites, where each sample pooled 3 plants. Temperature $=20^{\circ} \mathrm{C}(\mathrm{a}), 20.5^{\circ} \mathrm{C}(\mathrm{b}$, Experiment1), $18.5^{\circ} \mathrm{C}$ (Experiment2); 12L:12D light intensity $=190 \mu \mathrm{mol} / \mathrm{m}^{2} / \mathrm{s}(\mathrm{a}), 145$ $\mu \mathrm{mol} / \mathrm{m}^{2} / \mathrm{s}$ (b-n); $\mathrm{CO}_{2}=420 \mathrm{ppm}$. 
a

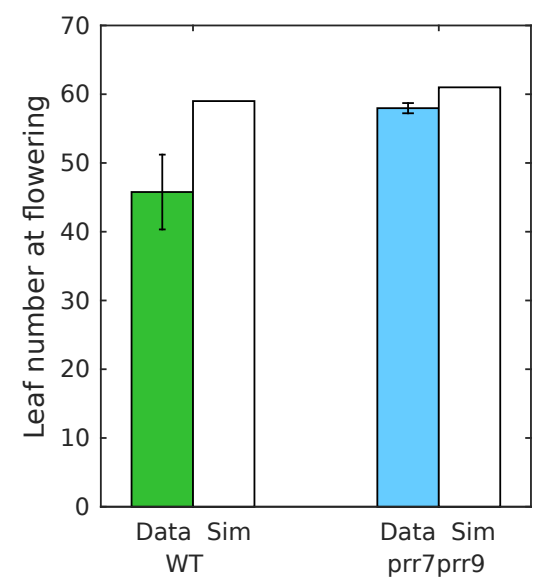

C

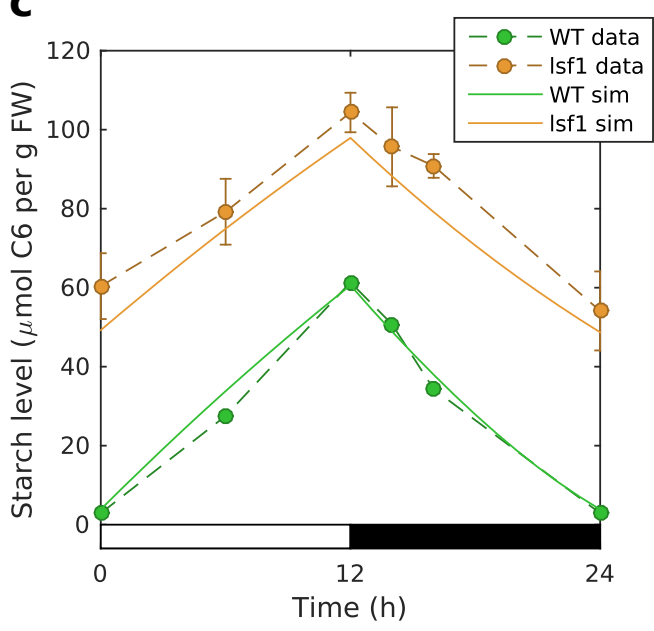

b

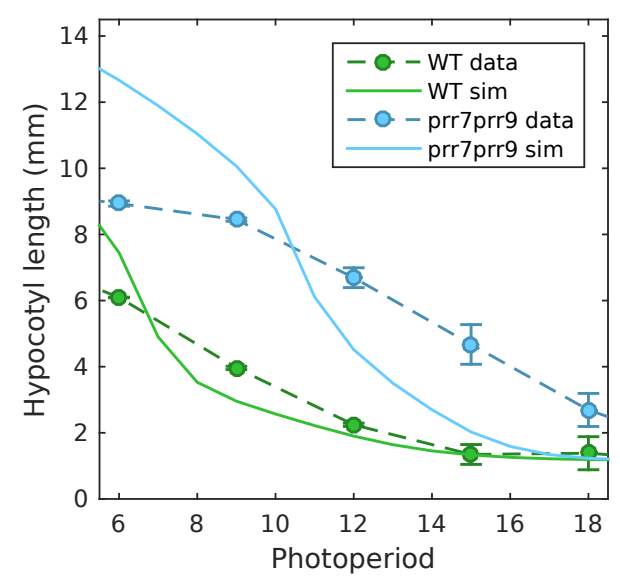

d

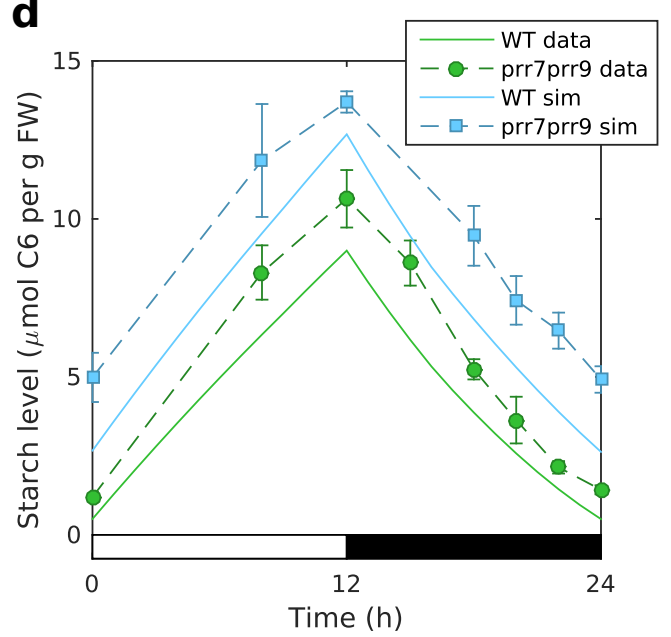

Extended Data Figure 1: Simulation of clock outputs.

(a) Simulated leaf number under short photoperiods for WT and prr7prr9, compared to data from (Nakamichi et al, 2007); (b) Simulated hypocotyl elongation in multiple photoperiods, compared to data of (Niwa et al, 2009); (c) starch levels in Isf1 under 12L:12D, compared to model simulations (Comparot-Moss et al, 2010); (d) Starch levels in prr7prr9 under 12L:12D, compared to model simulations (as in Fig 1c, plotted in absolute values). Model simulation (lines) and experimental data (symbols) of night-time starch level for Col (green line, filled circles) and prr7prr9 (blue line, open squares). These are the same data from Fig. 1c. Results are given as mean \pm SEM $(n=6)$. Temperature $=20^{\circ} \mathrm{C}$; light $=190 \mu \mathrm{mol} / \mathrm{m}^{2} / \mathrm{s}$; photoperiod $=12 \mathrm{hr}$ light: $12 \mathrm{hr}$ dark. 
a

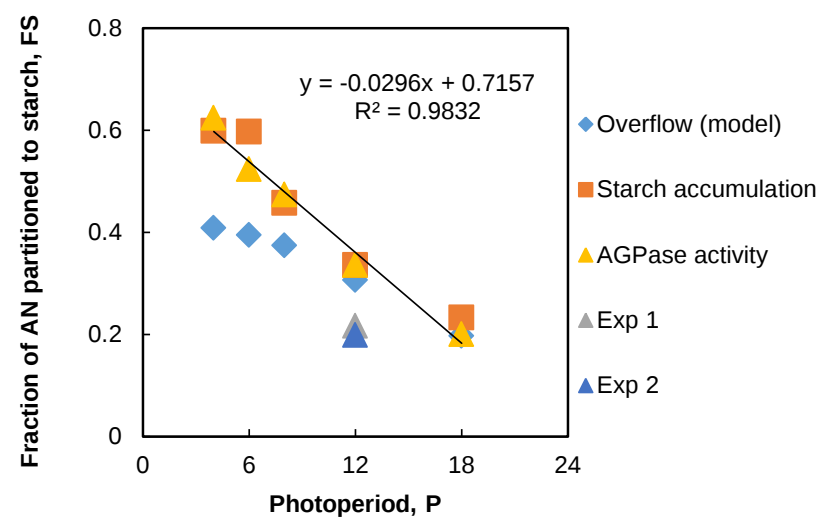

b

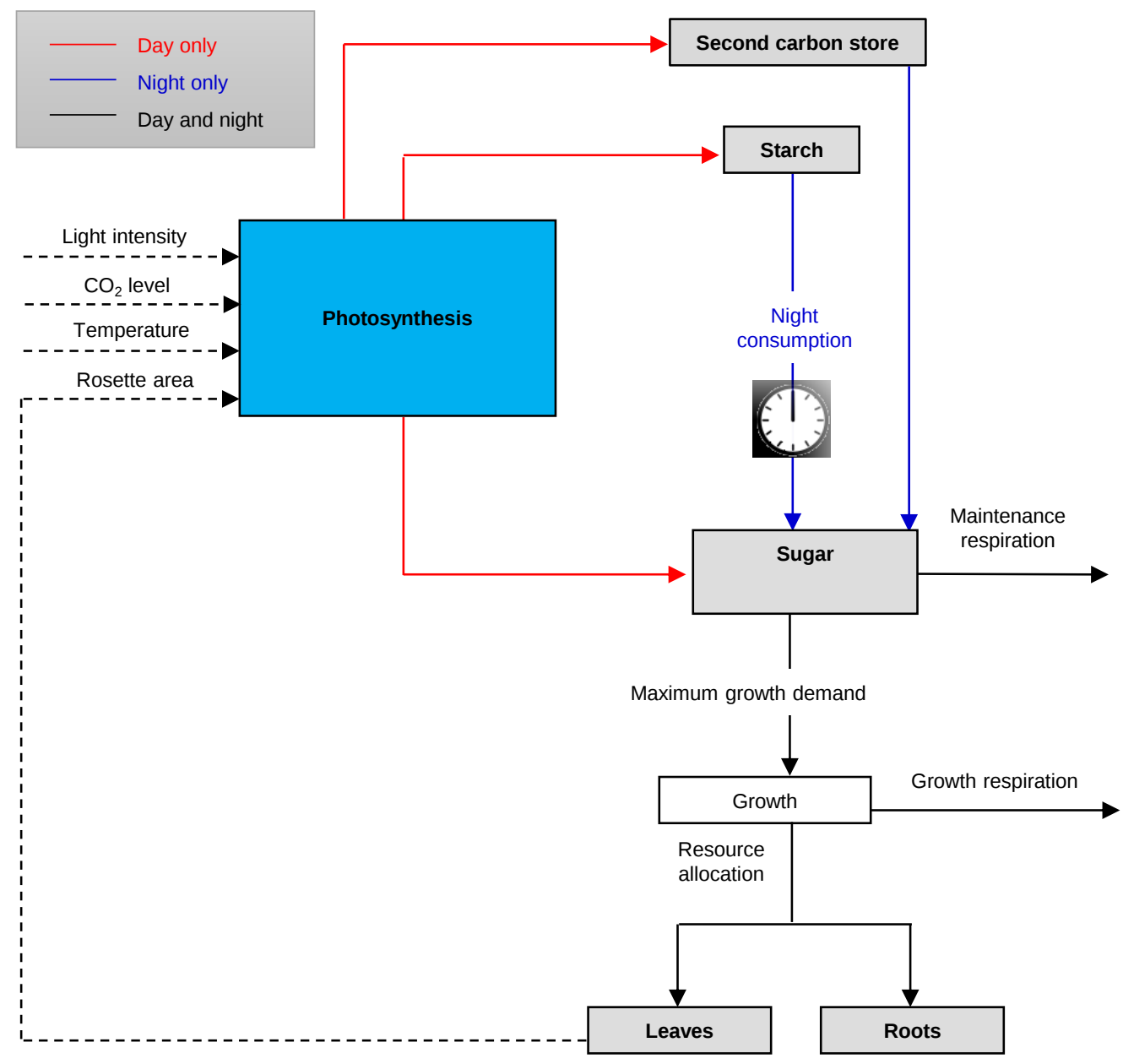

Extended Data Figure 2: Updating the Carbon Dynamic Model (CDM).

(a) The fractions of net assimilate partitioned to starch at different photoperiods, as simulated in the original CDM (FMv1) using the 'overflow' concept, calculated based on measured starch levels and calculated based on measured AGPase activity. The linear regression shown is for the AGPase activity series. The values used subsequently in the model for each experiment (Experiment 1, Experiment 2; Figure 3) are also shown. (b) Schematic of the new Carbon Dynamic Model (CDM). The second carbon store represents the total amount of malate and fumarate. The clock symbol represents the regulation of the rate of starch consumption by the circadian clock model. Dashed arrows indicate information or feedback input. 

available under aCC-BY 4.0 International license.
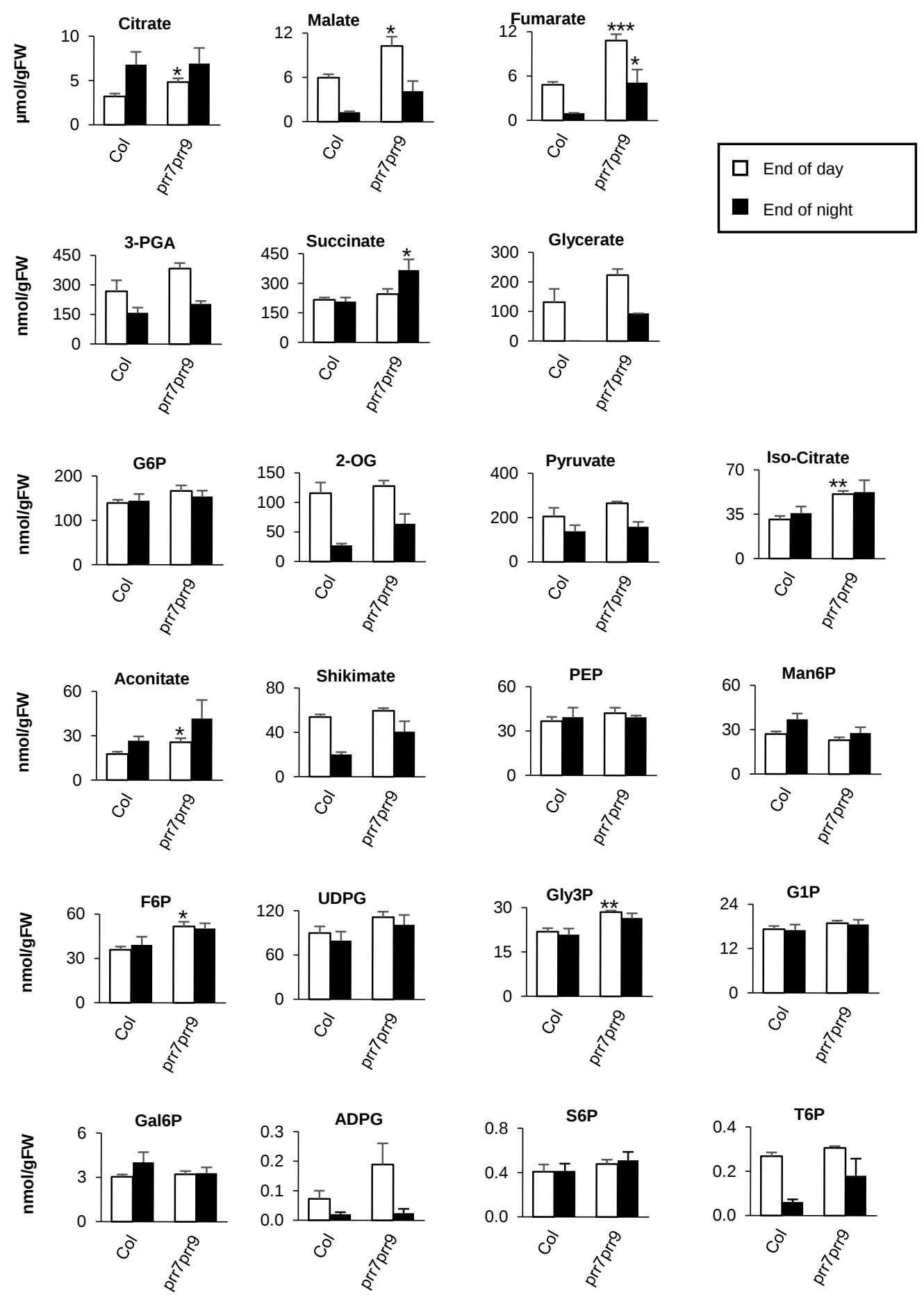

Extended Data Figure 3: Primary metabolites for Col and prr7prr9 mutant measured at the end of day and the end of night.

The results are given as the mean \pm SEM $(n=4)$. Each sample consisted of 5-7 pooled plants. Temperature $=20^{\circ} \mathrm{C}$ during the day and $18^{\circ} \mathrm{C}$ during the night; light $=160 \mu \mathrm{mol} / \mathrm{m}^{2} / \mathrm{s}$; photoperiod $=12 \mathrm{~h}$ light: $12 \mathrm{~h}$ dark. The $t$-test compared between Col and prr7prr9 (* $\mathrm{p}<0.05$; ** $\mathrm{p}<0.005$; *** $\mathrm{p}<0.001$ ). Note that the units given for citrate, malate, and fumarate are $\mu \mathrm{mol} / \mathrm{gFW}$, while the units given for the remaining metabolites are $\mathrm{nmol} / \mathrm{gFW}$. 


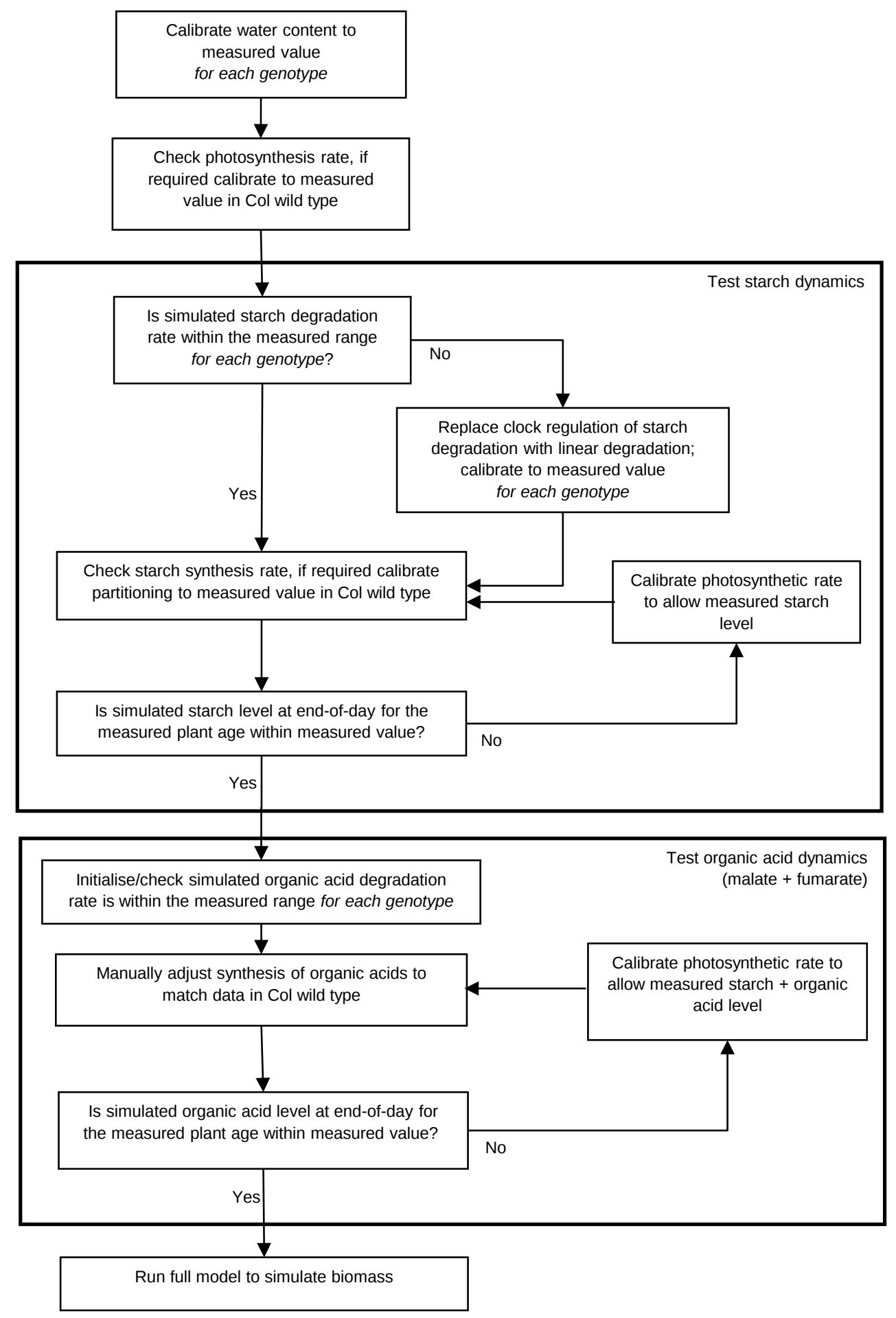

Extended Data Figure 4: Flow diagram of parameter calibration.

Parameters that could be directly or indirectly measured were adjusted in the illustrated sequence, to capture measured carbon dynamics and metabolite levels at specific time points. Once these were achieved, the model was simulated using the determined parameters to generate predictions for plant biomass. 
a

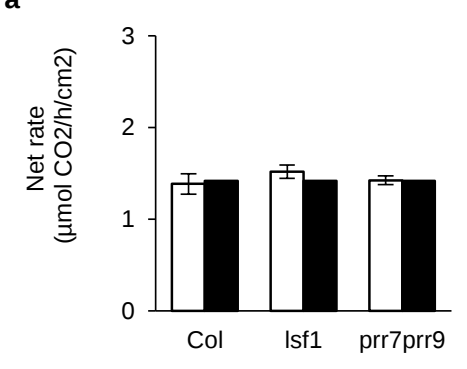

c

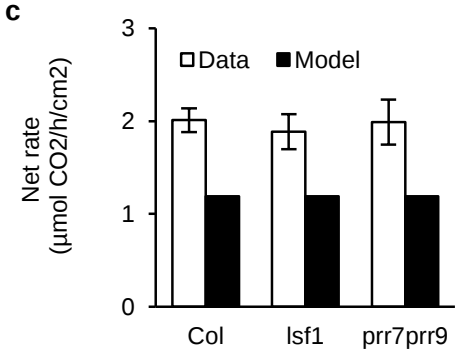

e

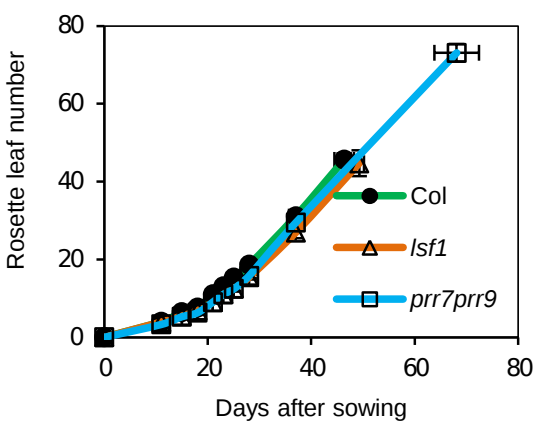

g

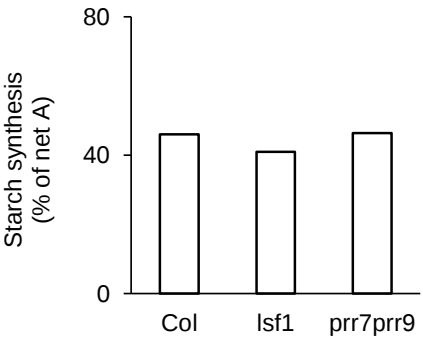

b
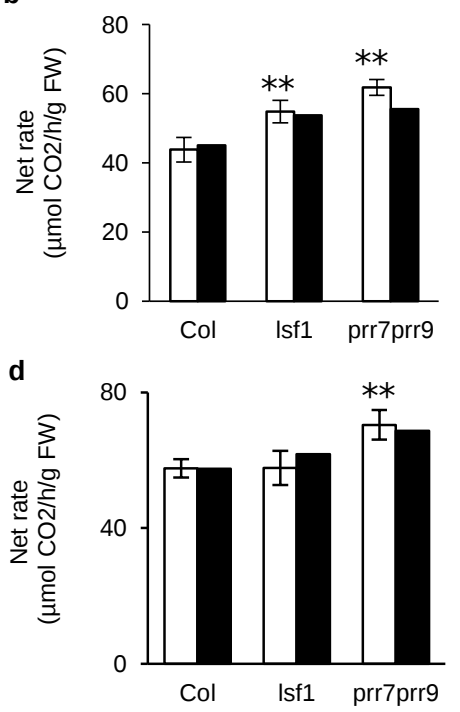

f
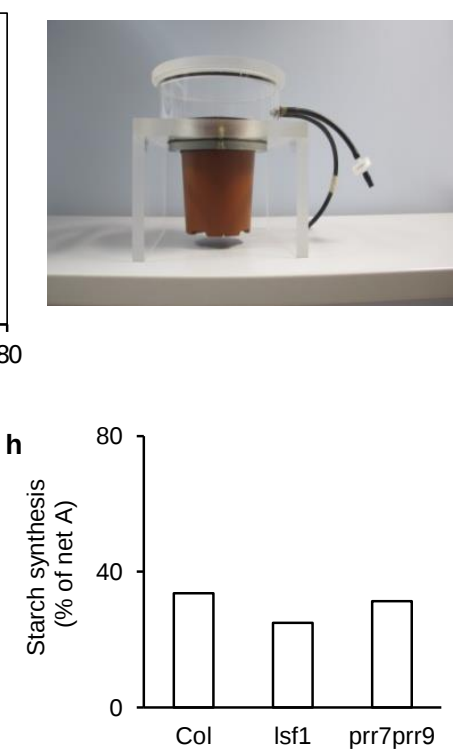

Extended Data Figure 5: Gas exchange measurement and leaf number of different mutants.

Net assimilation rate for Col, Isf1 and prr7prr9 in Experiment $1(\mathrm{a}, \mathrm{b})$ and Experiment 2 (c, d) expressed per unit rosette area (left column) and per gram fresh weight (right column). Data are shown as white bars while model simulations are shown as black bars. Data are given as mean \pm SD ( $n=5$ plants). There were no significant genotypic differences for net assimilation per unit area, thus similar rates were used in model simulations for all genotypes. However, net assimilation per unit fresh weight was significantly higher in Isf1 and prr7prr9 (** $p<0.005)$. Taking genotypic differences in water content into account was sufficient for the model to reproduce these results. (e) Rosette leaf number until flowering for Experiment 1. There was no significant difference in the flowering time of Isf1, while prr7prr9 was late-flowering. (f) The Plexiglass chamber used for gas exchange measurement. Starch synthesis as a percentage of net assimilation rate for Experiment 1 (g) and Experiment 2 (h). 

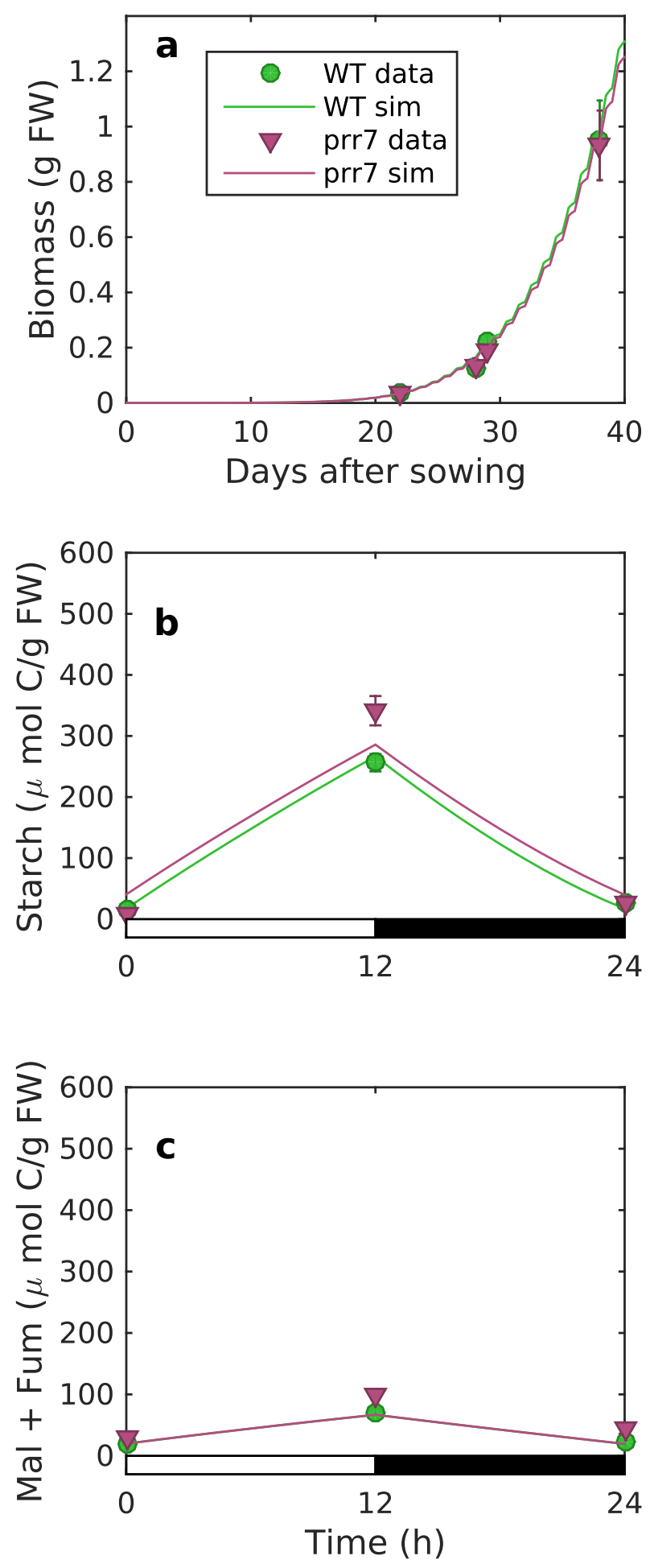

Extended Data Figure 6: Biomass and carbon status of prr7 mutants.

Measured (symbols) and simulated (lines) fresh weight (a), starch level (b), and malate and fumarate (c) for prr7 single mutant plants (purple) compared to wild type Col (green) in Experiment 1. Data for Col are identical to Fig.3a,3b. 

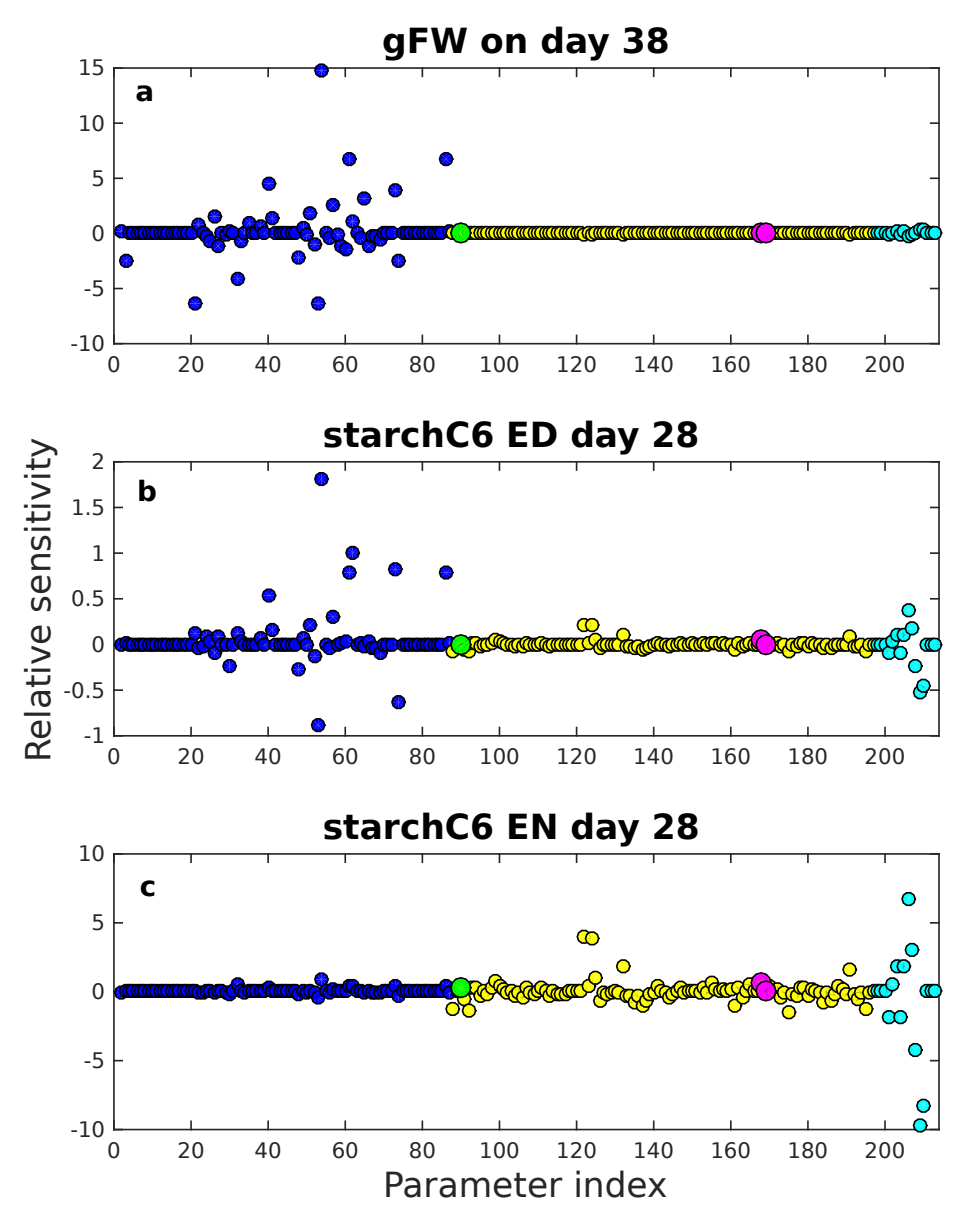

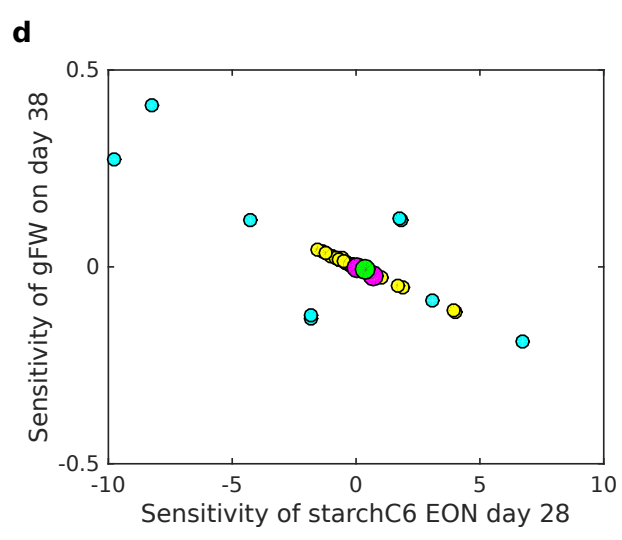

\begin{tabular}{|ll|}
\hline - & Core FM \\
$\circ$ & Clock module \\
$\circ$ & Starch module \\
O & PRR7 transcription \\
O & PRR9 transcription \\
\hline
\end{tabular}

Extended Data Figure 7: Parameter sensitivity overview.

Relative sensitivity of model outputs for each model parameter, coloured by the cognate sub-model (see legend). Sensitivities were calculated by simulating the model under 1\% perturbations of each parameter in turn. (a) Fresh Weight (FW); most sensitive parameter is $S_{\text {elec}}$, associated with electron transport, (b) starch level at end of day (ED); most sensitive parameter is $s_{\text {elec }}$ and (c) at end of night $(E N)$; most sensitive parameter is $k_{d T 1}$, the degradation rate of a putative inhibitor of starch turnover. Clock parameters mutated to simulate prr7prr9 double mutants are highlighted (see legend). (d) Comparison of sensitivity of FW and EON starch for parameters clock and starch module parameters, showing a predominant negative correlation: parameters that lower starch at the end of the night tend to increase fresh weight. Note that water content ( $w$, a directly measured parameter) is not shown due to high sensitivity. Sensitivities to changes in water content were 11.3, -10.1, and -10.1 for gFW, ED starch, and EN starch, respectively. 

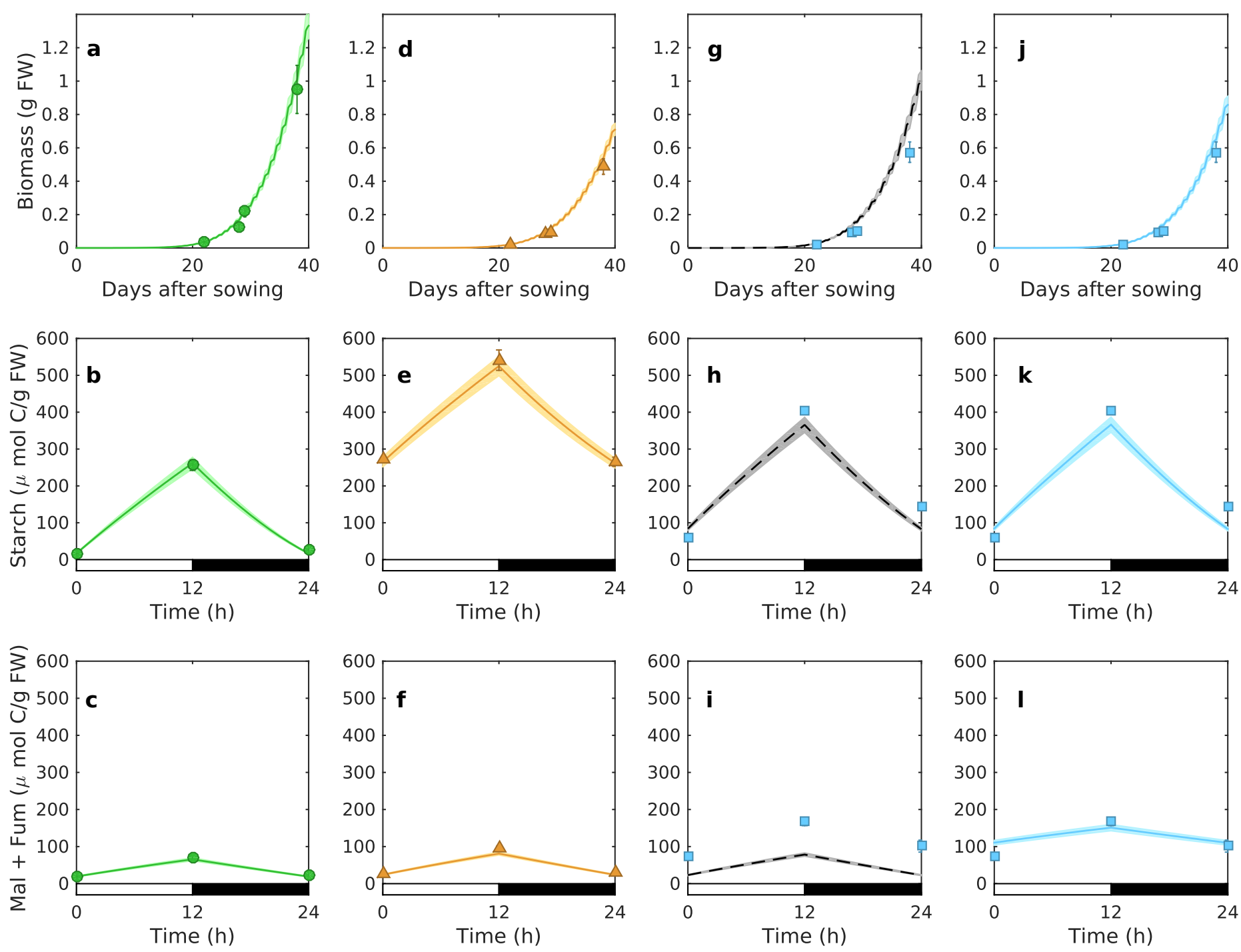

Extended Data Figure 8 : Model sensitivity to water content parameter.

Simulation of biomass and major metabolites using water content values plus and minus one standard deviation from the mean, for Experiment 1 in Figure 3. Model simulation (lines) andexperimental data (symbols) of fresh weight $(a, d, g, j)$, starch level $(b, e, h, k)$ and the total levelof malate and fumarate $(\mathrm{c}, \mathrm{f}, \mathrm{i}, \mathrm{I})$ for Col (a-c), Isf1 (d-f) and prr7prr9 (g-l). Dashed lines $(g-i)$ are model simulation for prr7prr9 that only considered starch defects, while full lines $(j-1)$ are model simulation that included both starch defects and inefficient use of malate and fumarate. Shaded regions indicate the values spanned by simulating water contents plus and minus one standard deviation from the mean. Data are given as mean \pm SD ( $n=5$ for biomass; $n=3$ for metabolites with each sample consisting of 3 pooled plants). Temperature $=20.5^{\circ} \mathrm{C}$; light $=145 \mu \mathrm{mol} / \mathrm{m}^{2} / \mathrm{s}$; photoperiod $=12 \mathrm{hr}$ light: 12 hr dark; $\mathrm{CO}_{2}=420$ ppm. 
bioRxiv preprint doi: https://doi.org/10.1101/105437; this version posted February 6, 2017. The copyright holder for this preprint (which was not certified by peer review) is the author/funder, who has granted bioRxiv a license to display the preprint in perpetuity. It is made available under aCC-BY 4.0 International license.

Extended Data Table 1 | New parameters and their calibrated values for each experiment, and model goodness-of-fit (cvRMSE)

\begin{tabular}{|c|c|c|c|c|c|c|}
\hline & & & Experiment 1 & & Experiment 2 & \\
\hline Genotypes & Parameters & $\begin{array}{l}\text { Parameter } \\
\text { names in } \\
\text { model file }\end{array}$ & Calibrated values & Remarks & Calibrated values & Remarks \\
\hline \multirow[t]{7}{*}{ Col (default) } & $\begin{array}{l}\text { Photosynthesis } \\
\text { efficiency }\end{array}$ & $\begin{array}{l}\text { photosyn_effi } \\
\text { ciency }\end{array}$ & 0.88 & $\begin{array}{c}\text { Calculated from } \\
\text { Col data }\end{array}$ & 0.79 & $\begin{array}{l}\text { Calculated from } \\
\text { Col data }\end{array}$ \\
\hline & $\begin{array}{l}\text { Starch synthesis } \\
\text { efficiency }\end{array}$ & ss_efficiency & 0.6 & $\begin{array}{c}\text { To fit the ED } \\
\text { Col starch level }\end{array}$ & 0.55 & $\begin{array}{c}\text { To fit the ED Col } \\
\text { starch level }\end{array}$ \\
\hline & Starch turnover* & sta_turnover & NA & $\begin{array}{c}\text { Simulated with } \\
\text { clock-regulated } \\
\text { starch model }\end{array}$ & 0.92 & $\begin{array}{c}\text { Calculated from } \\
\text { data }\end{array}$ \\
\hline & MF turnover & mf_use & 0.7 & $\begin{array}{c}\text { Calculated from } \\
\text { data }\end{array}$ & 0.6 & $\begin{array}{c}\text { Calculated from } \\
\text { data }\end{array}$ \\
\hline & MF synthesis & mf_syn_frac & 0.2 & $\begin{array}{c}\text { Calculated from } \\
\text { Col data }\end{array}$ & 0.2 & $\begin{array}{l}\text { Calculated from } \\
\text { Col data }\end{array}$ \\
\hline & Water content (\%) & w & 91.16 & Measured & 89.99 & Measured \\
\hline & cvRMSE (\%) & & 8.0 & & 16.0 & \\
\hline \multirow[t]{3}{*}{ Isf1 } & Starch turnover* & \begin{tabular}{|c|} 
sta_turnover \\
0.84 in \\
FMv1; clock- \\
regulated \\
model in \\
FMv2
\end{tabular} & $\begin{array}{c}\text { Clock-regulated } \\
\text { model } \\
k_{\mathrm{d}, \mathrm{S}}=10 \\
\mathrm{k}_{\mathrm{d}, \mathrm{T}, 2}=0.018\end{array}$ & $\begin{array}{c}\text { To fit the ED } \\
\text { and EN starch } \\
\text { levels }\end{array}$ & $\begin{array}{c}\text { sta_turnover } \\
0.56\end{array}$ & $\begin{array}{l}\text { To fit the ED and } \\
\text { EN starch levels }\end{array}$ \\
\hline & Water content (\%) & w & 89.43 & Measured & 89.25 & Measured \\
\hline & cvRMSE (\%) & & 13.7 & & 15.4 & \\
\hline \multirow[t]{5}{*}{ prr7/prr9 } & Starch turnover* & sta_turnover & NA & $\begin{array}{c}\text { Simulated clock } \\
\text { mutations } \\
\text { control starch } \\
\text { via clock- } \\
\text { regulated starch } \\
\text { model }\end{array}$ & 0.89 & $\begin{array}{l}\text { To fit the ED and } \\
\text { EN starch levels }\end{array}$ \\
\hline & Water content (\%) & w & 89.12 & Measured & 88.04 & Measured \\
\hline & MF turnover & mf_use & 0.25 & $\begin{array}{l}\text { To fit the ED } \\
\text { MF level }\end{array}$ & 0.21 & $\begin{array}{c}\text { To fit the ED MF } \\
\text { level }\end{array}$ \\
\hline & cVRMSE (\%) & & 41.1 & $\begin{array}{c}\text { Simulation with } \\
\text { only starch } \\
\text { defects }\end{array}$ & 44.8 & $\begin{array}{l}\text { Simulation with } \\
\text { only starch } \\
\text { defects }\end{array}$ \\
\hline & cvRMSE (\%) & & 15.1 & $\begin{array}{l}\text { Simulation with } \\
\text { defects in } \\
\text { starch, malate } \\
\text { and fumarate } \\
\text { consumption }\end{array}$ & 20.9 & $\begin{array}{l}\text { Simulation with } \\
\text { defects in starch, } \\
\text { malate and } \\
\text { fumarate } \\
\text { consumption }\end{array}$ \\
\hline
\end{tabular}

*The sta_turnover parameter in FMv1 (value 0.84) is not used in FMv2, because starch degradation rate is computed by the clock-regulated starch model. Where prr7prr9 showed a mild starch phenotype, in experiment 2, sta_turnover was calibrated as described in Extended Data Fig.4; the same model was used to compare all genotypes in the experiment. 


\section{Supplementary methods - modelling}

\section{Contents}

Supplementary methods - modelling. 1

1. Updating the circadian clock, starch, and photoperiod response models 1

1.1 Photoperiod response model 1

1.2 Circadian control of starch turnover.

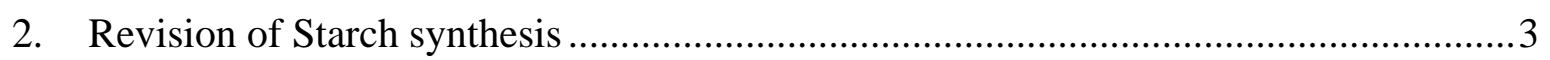

3. Addition of carbon pool for malate and fumarate.......................................................

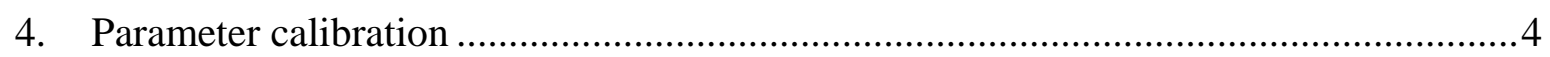

5. Modelling protein synthesis, compared to literature data ...........................................5

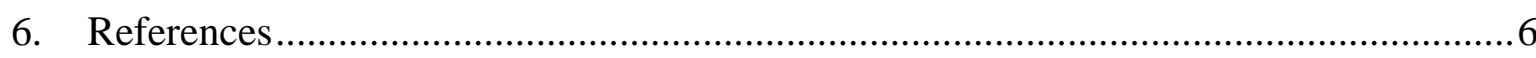

\section{Updating the circadian clock, starch, and photoperiod response models}

\subsection{Photoperiod response model}

The circadian clock controls the timing of flowering by regulating the expression of the $F T$ gene through the photoperiod pathway. The photoperiod response was previously modelled in the Arabidopsis Framework Model version 1 (FMv1) ${ }^{1}$ by including the model from Salazar et al $2009^{2}$. However, this model includes an older circadian clock model ${ }^{3}$ that does not explicitly represent the relevant clock components $P R R 9$ and $P R R 7$. We therefore replaced the Salazar model with our most recent, Seaton-Smith model of the photoperiod pathway ${ }^{4}$. This brings several advantages. First, the Seaton-Smith model includes additional understanding of the photoperiod response mechanism, such as the regulation of $\mathrm{CO}$ protein stability by FKF1 ${ }^{5}$. Second, it is based upon the same circadian clock model ${ }^{6}$ as the clockstarch model that we introduce in Section 1.2, below. Third, the clock model includes PRR9 and $P R R 7$, allowing explicit simulation of the prr9prr7 mutation (see section 1.2.2). Fourth, the Seaton-Smith model represents circadian regulation of hypocotyl elongation via the PIF transcription factors, allowing the FMv2 to represent this canonical clock phenotype.

As in the Salazar and FMv1 models, the photoperiod response model in the FMv2 interacts with the phenology model through the control of FT transcript expression. The important characteristic is 'FTarea', the integrated FT level over the course of a $24 \mathrm{~h}$ day. FTarea controls the Photoperiod component of the phenology model through the expression:

$$
\text { Photoperiod }=a+b\left[\frac{c^{n}}{c^{n}+\text { FTarea }^{n}}\right]
$$

In order to utilise this connection with the new circadian clock model, the parameters $b, c$ and $n$ were chosen so that this function matched the original photoperiod function given by Chew et al $2012^{7}$, as was done previously for the connection from the older clock model in Chew et al $2014^{1}$. 


\subsection{Circadian control of starch turnover}

The circadian clock controls the rate of starch degradation during the night in light:dark cycles ${ }^{8,9}$. The molecular mechanisms responsible for this control have not been identified, but our recent work identified simple, plausible mechanisms ${ }^{10}$. These were formalised in mathematical models that were evaluated by comparison to a wide range of experimental data (e.g. the change in starch turnover when dusk arrives $\sim 4$ hours early). In Seaton et al $2014{ }^{10}$, three models were described in detail, named Model Variants 1, 2 and 3. Of these, Model Variants 2 and 3 provided the best match to experimental data, while Model Variant 1 was shown to have several limitations. Since Model Variants 2 and 3 provided quantitatively similar predictions over a range of conditions, and Model Variant 2 is simpler (6 fewer parameters and 2 fewer regulatory links from the circadian clock), we chose to integrate Model 2 with the FMv2.

\subsubsection{Starch model structure}

In order to incorporate this control of starch turnover with the FM, we treat the starch component $S$ as a measure of starch concentration (rather than absolute quantity per plant). Thus, this is taken as:

$$
S(t)=r \frac{C_{\text {starch }}(t)}{C_{\text {shoot }}(t)}
$$

Where $S(\mathrm{t})$ is starch concentration variable used in the model of starch turnover, $C_{\text {starch }}(\mathrm{t})$ and $C_{\text {shoot }}(\mathrm{t})$ are the carbon in starch and in the shoot biomass respectively, and $r$ is a scaling factor used to bring $S(\mathrm{t})$ to a similar range of concentrations to those used in the original model construction ${ }^{10}$. Note, the control of starch synthesis by the species $Y$ is disregarded, as starch synthesis is modelled as a fixed fraction of photoassimilate (see Section 1.2).

This model runs on an hourly basis throughout the day, but controls starch turnover only during the night. The starch concentration (i.e. $S(t)$ ) is calculated at the start of the timestep, and the change in starch levels by the end of the hour is then given by:

$$
\Delta C_{\text {starch }}(t)=\frac{C_{\text {shoot }}(t) \Delta S(t)}{r}
$$

Where $\Delta S(t)$ denotes the change in starch concentration across the hour of simulation. Total starch carbon at the following timepoint is then updated according to:

$$
C_{\text {starch }}(t+1)=C_{\text {starch }}(t)+\Delta C_{\text {starch }}(t)
$$

\subsubsection{Simulating Isf1 and prr7prr9 mutant genotypes}

In order to simulate the circadian clock mutant $\operatorname{prr} 7 p r r 9$, we set to 0 the clock parameters $q_{3}$, $n 4, n 7, n 8$, and $n 9$, which control the multiple aspects of the transcription rate of $P R R 7$ and PRR9. Model simulations predicted $\sim 70 \%$ turnover of starch in the mutant, in agreement with experimental data (Fig. 3a and Extended Data Figure 1d).

All other parameter values were calibrated as described in Section 4, below (Extended Data Fig.4), and are shown in Extended Data Table 1. The starch degradation rate parameter in FMv1 (sta_turnover) is not used in FMv2, because the starch degradation rate is computed by 
the clock-regulated starch model. In order to simulate the $l_{s f} f$ mutant, the parameters $k_{d, S}$ and $k_{d, T, 2}$ in this model were set to 10 and 0.018 , respectively, calibrating simulated starch to our experimental data. This allowed the model to match the experimentally observed starch turnover in our experiment 1 (Fig. 3g) and in literature data ${ }^{11}$ (Extended Data Figure 1c). Where prr7prr9 showed a mild starch phenotype in experiment 2, sta_turnover was calibrated as described in Extended Data Fig.4; the same model was used to compare all genotypes in the experiment.

\section{Revision of Starch synthesis}

In the original Carbon Dynamic Model (CDM) ${ }^{1,12}$, starch is synthesised at a rate that is the sum of a baseline rate and an 'overflow' rate. The baseline rate is a fixed proportion of the photoassimilate. The rest of the photoassimilate is first converted into soluble sugars which are used for growth and respiration. As growth demand is limited to a maximum value, any excess photoassimilate is converted into starch, through the 'overflow' rate.

Our previous work 1,13 showed that the 'overflow' mechanism is not always applicable, especially when plants are grown in short-day conditions (Figure 1e). Results suggested that starch is synthesised at a photoperiod-dependent fixed rate that is much higher than the baseline, and any excess photoassimilate remains as sugars. This ensures that plants store sufficient starch to last the night. We therefore re-routed the carbon flow based on this finding.

To determine the photoperiod-dependent starch synthesis rate, we first calculated the fraction of measured net assimilate partitioned to starch using our previous data ${ }^{13}$ and the equation below:

$$
F_{S}=\frac{S_{E D}-S_{E N}}{A_{N} \times P}
$$

where

$$
\begin{array}{lll}
F_{S} & = & \text { Fraction partitioned to starch } \\
S_{E D} & = & \text { Starch level at ED } \\
S_{E N} & = & \text { Starch level at EN } \\
A_{N} & = & \text { Net assimilation rate per hour } \\
P & = & \text { Photoperiod }
\end{array}
$$

It has been reported that under low light conditions, most of the flux control through the pathway of starch synthesis resides in the reaction catalysed by AGPase ${ }^{14}$. Since most lab experiments are conducted under low light, we therefore also tested the relation between the fraction partitioned to starch and AGPase activity. If the total amount of starch accumulated over the light period is proportional to daily AGPase activity (averaged between ED and EN), the fraction is given by: 


$$
F_{S}(P)=\frac{k\left[\text { AGPase }_{\text {average }}(P)\right]}{A_{N} \times P}
$$

where $k$ is the proportional constant. We determined the value of $k$ using data from 12 -hr photoperiod as the reference. We found a strong linear relation between the fraction of measured net assimilate and photoperiod (Extended Data Figure 2). This relation is therefore used in the FMv2 to determine starch synthesis rate, StaSyn, as follows:

$$
\text { StaSyn }=A_{N} \times(-0.0296 P+0.7157)
$$

\section{Addition of carbon pool for malate and fumarate}

Malate and fumarate can be interconverted in the tricarboxylic acid cycle, so they are considered together in a single pool. The dynamics of this pool is modelled in a manner similar to starch except for the regulation of degradation rate by the clock. In the daytime, a fixed proportion of the photoassimilate is converted to starch, malate and fumarate, while sugar level is allow to fluctuate depending on the carbon excess. At night, malate and fumarate are consumed with a linear rate, while starch degradation rate is controlled by the clock sub-model (see Extended Data Figure 2 and Section 1.2). For simplicity, we model a direct conversion of carbon from malate and fumarate into sugar at night, omitting the intermediate metabolic reactions.

\section{Parameter calibration}

Results in our previous studies ${ }^{1,13}$ suggested that carbon dynamics in plants are flexible and plants adjust processes like photosynthesis, starch synthesis and starch degradation rate depending on the environment. The aims of our study were to test if the dynamics of the different carbon pools can be quantitatively balanced over the timescale of vegetative growth, and how genetic regulation that modifies these dynamics affects plant growth. It is therefore necessary that the model first matches quantitatively the carbon pool data for wild-type plants as the reference genotype in each study. After accounting for environmental effects on all genotypes through the wild-type data, discrepancies between model simulations and data for the mutants can be attributed to genetic effects. To achieve this, we calibrated the following to the Col data (workflow illustrated in Extended Data Figure 4; parameter values in Extended Data Table 1):

$>$ photosynthesis rate was adjusted by introducing an efficiency factor relative to the default

$>$ starch synthesis rate was adjusted by introducing an efficiency factor relative to the default

Starch turnover was simulated by the clock-controlled starch submodel (Section 1.2), which reproduced experimental measurement of percentage turnover in most cases. In cases where the phenotype of starch degradation was too mild and could not be explained by the starch submodel, we used a linear degradation rate as in the previous model version (FMv1) to 
reproduce the turnover. We then iteratively tuned starch synthesis and photosynthesis rates to match the measured end-of-day level (Extended Data Table 1).

We next calibrated the parameter values for the new carbon pool that represents malate and fumarate (MF) using Col data as follows:

$>$ The initial level of this pool was set as 0.4 of initial starch level, based on the ratio measured in the literature ${ }^{15}$

$>$ MF synthesis was set as a fixed fraction of starch synthesis

$>$ MF turnover was set as the fraction of dusk level consumed

Wherever possible, we used parameter values measured or calculated from our data. Mutants were simulated by changing the values of genotype-specific parameters as listed in Extended Data Table 1, notably the water content.

In each experiment, we did not find genotypic differences in photosynthesis when expressed per unit area, but there was a general increase in photosynthesis in $\operatorname{prr} 7 \mathrm{prr} 9$ when expressed per gram fresh weight (Extended Data Figure 5). Even though we used the same photosynthesis efficiency for all genotypes, we found that the model could reproduce this increase due to the lower water content measured in prr7prr9. This suggested the importance of including water content as a genotype-specific parameter in our model, since metabolites are measured per unit fresh weight.

As expected, we found variation in photosynthesis efficiency between experiments. In particular, the photosynthesis per unit area was higher for all genotypes in Experiment 2. As a result, the model underestimated these, but reproduced the values when expressed per unit fresh weight, suggesting a difference in the specific leaf area.

\section{Modelling protein synthesis, compared to literature data}

The biomass prediction in the FMv2 implies minimal budgets for the nutrient constituents of biomass, which are effectively predictions that can be compared to published experimental data. For example, ${ }^{13} \mathrm{CO}_{2}$ labelling has allowed quantification of the relative rates of protein synthesis in the light and dark during light:dark cycles ${ }^{16}$, and of rates of protein turnover ${ }^{17}$.

The model does not include protein as a distinct component of the synthesised biomass. However, since the protein fraction of biomass is relatively constant across the course of a day (for example, see Pyl et al $2012{ }^{18}$ ), and protein turnover has been measured, it is possible to calculate an implied rate of protein synthesis for a given model simulation (as done experimentally in Ishihara et al $2015^{17}$ ). In particular:

$$
\operatorname{ProtSyn}(t)=(G r(t)+\text { Turn }) \text { Prot }
$$

where $\operatorname{ProtSyn}(t)$ is the calculated rate of protein synthesis at time $t$, in units of gProtein gFW $^{-}$ ${ }^{1} \mathrm{~h}^{-1} . G r(t)$ is the relative growth rate $(=(\operatorname{Biomass}(t)-\operatorname{Biomass}(t-1)) / \operatorname{Biomass}(t))$, in units of $\mathrm{hr}^{-}$ 1. Turn is the rate of protein turnover, measured as $0.0014 \mathrm{hr}^{-1}$ (average of measurements by Pulse-Chase labelling ${ }^{17}$ ). Prot is the protein content, measured as 0.0169 gProtein gFW $^{-1}$ in Ishihara et al $2015^{17}$.

Simulating the conditions used in Ishihara et al $2015{ }^{17}$ for wild-type plants shows that carbon biomass growth rates in the model predict a 3.3-fold increase in the rate of protein synthesis 
during the day, compared to during the night. This is in excellent agreement with experimental data which showed a 3.1-fold increase ${ }^{17},{ }^{18}$.

\section{References}

1 Chew, Y. H. et al. Multiscale digital Arabidopsis predicts individual organ and wholeorganism growth. $P$ Natl Acad Sci USA 111, E4127-E4136, doi:10.1073/pnas.1410238111 (2014).

2 Salazar, J. D. et al. Prediction of Photoperiodic Regulators from Quantitative Gene Circuit Models. Cell 139, 1170-1179, doi:10.1016/j.cell.2009.11.029 (2009).

3 Locke, J. C. W. et al. Extension of a genetic network model by iterative experimentation and mathematical analysis. Mol Syst Biol 1, doi:10.1038/Msb4100018 (2005).

4 Seaton, D. D. et al. Linked circadian outputs control elongation growth and flowering in response to photoperiod and temperature. Mol Syst Biol 11 (2015).

5 Song, Y. H., Smith, R. W., To, B. J., Millar, A. J. \& Imaizumi, T. FKF1 conveys timing information for CONSTANS stabilization in photoperiodic flowering. Science 336, 1045-1049, doi:10.1126/science.1219644(2012).

6 Pokhilko, A. et al. The clock gene circuit in Arabidopsis includes a repressilator with additional feedback loops. Mol Syst Biol 8, doi:10.1038/Msb.2012.6 (2012).

7 Chew, Y. H. et al. An augmented Arabidopsis phenology model reveals seasonal temperature control of flowering time. New Phytol 194, 654-665, doi:10.1111/j.14698137.2012.04069.x (2012).

8 Scialdone, A. et al. Arabidopsis plants perform arithmetic division to prevent starvation at night. Elife 2, doi:10.7554/eLife.00669 (2013).

9 Graf, A., Schlereth, A., Stitt, M. \& Smith, A. M. Circadian control of carbohydrate availability for growth in Arabidopsis plants at night. P Natl Acad Sci USA 107, 94589463, doi:10.1073/pnas.0914299107 (2010).

10 Seaton, D. D., Ebenhoh, O., Millar, A. J. \& Pokhilko, A. Regulatory principles and experimental approaches to the circadian control of starch turnover. $J$ R Soc Interface 11, doi:10.1098/Rsif.2013.0979 (2014).

11 Comparot-Moss, S. et al. A Putative Phosphatase, LSF1, Is Required for Normal Starch Turnover in Arabidopsis Leaves. Plant Physiol 152, 685-697, doi:10.1104/pp.109.148981 (2010).

12 Rasse, D. P. \& Tocquin, P. Leaf carbohydrate controls over Arabidopsis growth and response to elevated CO2: an experimentally based model. New Phytol 172, 500-513, doi:10.1111/j.1469-8137.2006.01848.x (2006).

13 Sulpice, R. et al. Arabidopsis Coordinates the Diurnal Regulation of Carbon Allocation and Growth across a Wide Range of Photoperiods. Mol Plant 7, 137-155, doi:10.1093/mp/sst127 (2014). 
14 Neuhaus, H. E. \& Stitt, M. Control Analysis of Photosynthate Partitioning - Impact of Reduced Activity of Adp-Glucose Pyrophosphorylase or Plastid Phosphoglucomutase on the Fluxes to Starch and Sucrose in Arabidopsis-Thaliana (L) Heynh. Planta 182, 445-454 (1990).

15 Chia, D. W., Yoder, T. J., Reiter, W. D. \& Gibson, S. I. Fumaric acid: an overlooked form of fixed carbon in Arabidopsis and other plant species. Planta 211, 743-751 (2000).

16 Pal, S. K. et al. Diurnal Changes of Polysome Loading Track Sucrose Content in the Rosette of Wild-Type Arabidopsis and the Starchless pgm Mutant. Plant Physiol 162, 1246-1265, doi:10.1104/pp.112.212258 (2013).

17 Ishihara, H., Obata, T., Sulpice, R., Fernie, A. R. \& Stitt, M. Quantifying Protein Synthesis and Degradation in Arabidopsis by Dynamic (CO2)-C-13 Labeling and Analysis of Enrichment in Individual Amino Acids in Their Free Pools and in Protein (vol 168, pg 74, 2015). Plant Physiol 168, 1179-1179, doi:10.1104/pp.15.00771 (2015).

18 Pyl, E. T. et al. Metabolism and Growth in Arabidopsis Depend on the Daytime Temperature but Are Temperature-Compensated against Cool Nights. Plant Cell 24, 2443-2469, doi:10.1105/tpc.112.097188 (2012). 